\title{
Anomalous decay rate of quasinormal modes in Schwarzschild-dS and Schwarzschild-AdS black holes
}

\author{
Almendra Aragón, ${ }^{a}$ P.A. González, ${ }^{a}$ Eleftherios Papantonopoulos ${ }^{b}$ and Yerko Vásquez ${ }^{c}$ \\ ${ }^{a}$ Facultad de Ingeniería y Ciencias, Universidad Diego Portales, \\ Avenida Ejército Libertador 441, Casilla 298-V, Santiago, Chile \\ ${ }^{b}$ Physics Division, National Technical University of Athens, \\ 15780 Zografou Campus, Athens, Greece \\ ${ }^{c}$ Departamento de Física y Astronomía, Facultad de Ciencias, Universidad de La Serena, \\ Avenida Cisternas 1200, La Serena, Chile \\ E-mail: almendra.aragon@mail.udp.cl, pablo.gonzalez@udp.cl, \\ lpapa@central.ntua.gr, yvasquez@userena.cl
}

ABSTRACT: Recently an anomalous decay rate of the quasinormal modes of a massive scalar field in Schwarzschild black holes backgrounds was reported in which the longestlived modes are the ones with higher angular number, for a scalar field mass smaller than a critical value, while that beyond this value the behaviour is inverted. In this work, we extend the study to other asymptotic geometries, such as, Schwarzschild-de Sitter and Schwarzschild-AdS black holes. Mainly, we found that such behaviour and the critical mass are present in the Schwarzschild-de Sitter background. Also, we found that the value of the critical mass increases when the cosmological constant increases and also when the overtone number is increasing. On the other hand, despite the critical mass is not present in Schwarzschild-AdS black holes backgrounds, the decay rate of the quasinormal modes always exhibits an anomalous behaviour.

KEYwords: Black Holes, Classical Theories of Gravity

ARXIV EPRINT: 2004.09386 


\section{Contents}

1 Introduction 1

2 Scalar perturbations $\quad 3$

2.1 Numerical analysis. Schwarzchild-de Sitter black holes 4

2.1.1 Photon sphere modes 5

2.1.2 dS modes 8

2.2 Numerical analysis. Schwarzchild-AdS black holes 9

3 Analysis using the WKB method 11

4 Conclusions $\quad 14$

$\begin{array}{ll}\text { A Accuracy of the numerical method } & 16\end{array}$

B Numerical values. Schwarzschild-de Sitter black hole with $M^{2} \Lambda=0.04 \quad 17$

C Numerical values. Schwarzschild-de Sitter black holes with $M^{2} \Lambda=0.1121$

D Numerical values. Schwarzschild-AdS black holes 22

\section{Introduction}

The quasinormal modes (QNMs) and quasinormal frequencies (QNFs) [1-6] have recently acquired great interest due to the detection of gravitational waves [7]. Despite the detected signal is consistent with the Einstein gravity [8], there are possibilities for alternative theories of gravity due to the large uncertainties in mass and angular momenta of the ringing black hole [9]. The QNMs and QNFs give information about the stability of matter fields that evolve perturbatively in the exterior region of a black hole without backreacting on the metric. Also, the QNMs are characterized by a spectrum that is independent of the initial conditions of the perturbation and depends on the black hole parameters and probe field parameters, and on the fundamental constants of the system. The QNM infinite discrete spectrum consists of complex frequencies, $\omega=\omega_{R}+i \omega_{I}$, in which the real part $\omega_{R}$ determines the oscillation timescale of the modes, while the complex part $\omega_{I}$ determines their exponential decaying timescale (for a review on QNM modes see $[3,6]$ ).

The QNFs have been calculated by means of numerical and analytical techniques; some well known numerical methods are: the Mashhoon method, Chandrasekhar-Detweiler method, WKB method, Frobenius method, method of continued fractions, Nollert, asymptotic iteration method (AIM) and improved AIM, among others. In the case of gravitational perturbations it was found that for the Schwarzschild and Kerr black hole backgrounds the 
longest-lived modes are always the ones with lower angular number $\ell$. This is expected in a physical system because the more energetic modes with high angular number $\ell$ would have faster decaying rates. In the case of a massive probe scalar field it was found [10-13], at least for the overtone $n=0$, that if we have a light scalar field, then the longest-lived quasinormal modes are those with a high angular number $\ell$, whereas for a heavy scalar field the longest-lived modes are those with a low angular number $\ell$. This behaviour can be understood because for the case of massive scalar field even if its mass is small its fluctuations can maintain the quasinormal modes to live longer even if the angular number $\ell$ is large. This anomalous behaviour is depending on whether the mass of the scalar field exceeds a critical value or not. This anomalous decay rate for small mass scale of the scalar field was recently discussed in [14].

Extensive study of QNMs of black holes in asymptotically flat spacetimes have been performed for the last few decade mainly due to the potential astrophysical interest. Considering the case when the black hole is immersed in an expanding universe, the QNMs of black holes in de Sitter (dS) space have been investigated [15-20]. The AdS/CFT correspondence $[21,22]$ stimulated the interest in calculating the QNMs and QNFs of black holes in anti-de Sitter (AdS) spacetimes. It was shown in [23] that this principle leads to a correspondence of the QNMs of the gravity bulk to the decay of perturbations in the dual conformal field theory, and the QNFs were also studied in ref. [24].

The aim of this work is to study the propagation of scalar fields in the SchwarzschilddS and Schwarzschild-AdS black hole backgrounds in order to see if there is an anomalous decay rate of quasinormal modes. The motivation for this study is to study the effect of the presence of the cosmological constant on the anomalous decay. For gravitational perturbations in Schwarzschild de Sitter black holes the longest-lived modes are always the one with lower angular number $\ell$ [25]. In the case of a massive probe scalar field, as we already discussed, a different behaviour in the Schwarzschild background was observed, here a mass scale is introduced by the probe scalar field. However, the cosmological constant is introducing another scale and it is interesting to see what is the effect on the anomalous behaviour of the competition of the two scales for both positive and negative cosmological constant. We carry out this study by using the pseudospectral Chebyshev method [26] which is an effective method to find high overtone modes [27-31].

The gravitational QNMs of Schwarzschild-de Sitter black hole were studied in [25, 33, 34]. The QNMs of a probe scalar field for this geometry were calculated in [35] by using the sixth order WKB formula and the approximation by the Pöschl-Teller (P-T) potential. Also, it was shown the frequencies all have a negative imaginary part, which means that the propagation of scalar fields is stable in this background. The presence of the cosmological constant leads to decrease of the real oscillation frequency and to a slower decay, and high overtones was studied in ref. [36]. Also, a novel infinite set of purely imaginary modes was found [37], which depending on the black hole mass may even be the dominant mode.

In the case of a massless scalar field in the background of a Schwarzschild-dS black hole we find two types of QNMs, a family of complex quasinormal modes which are well described by the WKB formula (photon sphere modes) and a family of pure imaginary ones, which are closely related to the de Sitter horizon (dS modes) [38]. These modes 
have a different behaviour as the cosmological constant is changing. First of all for the complex modes all the frequencies have a negative imaginary part, which means that the propagation of scalar field is stable in this background. However the presence of a larger cosmological constant leads to decrease the real oscillation frequency and to a slower decay. On the contrary in the case of pure imaginary modes we find the cosmological constant leads to a fast decay, when it increases, that is, contrary to the complex QNFs.

In the case of a massive scalar field in the background of a Schwarzschild-dS black hole we find that the imaginary part of the photon sphere QNFs has an anomalous behaviour for a scalar field mass less than a critical mass, i.e, the absolute value of the imaginary part decay when the angular harmonic numbers $\ell$ increase; and for a scalar field mass greater than the critical mass the behavior is inverted, i.e, the longest-lived modes are always the ones with higher angular number. The critical mass corresponds to the value of the scalar field mass where the behavior of the decay rate of the QNMs is inverted and can be obtained from the condition $\operatorname{Im}(\omega)_{\ell}=\operatorname{Im}(\omega)_{\ell+1}$ in the eikonal limit, that is when $\ell \rightarrow \infty$. Additionally, we find that as the value of the cosmological constant increases the value of the critical mass also increases.

On the other hand, we do not observe an anomalous decay for the dS modes. We also show that the dS modes can acquire a real part which depends on the scalar fields mass. The QNMs of massive scalar fields in the Schwarzschild-dS black hole background were studied in [39] showing that there a lower limit of the scalar mass which allows the waves with QNFs to propagate at infinity. In the case of Schwarzschild-AdS black hole background we find a faster decay when the mass of the scalar field increases and when the angular harmonic numbers $\ell$ decrease.

The manuscript is organized as follows: in section 2 , we study the scalar field stability by calculating the QNFs of scalar perturbations numerically of a massless and massive scalar field in the background of Schwarzschild-dS and Schwarzschild-AdS black hole background by using the pseudospectral Chebyshev method. In section 3 we perform an analysis using the WKB method to get some analytical insight. We conclude in section 4.

\section{Scalar perturbations}

The Schwarzschild-(dS)AdS black holes are maximally symmetric solutions of the equations of motion that arise from the action

$$
S=\frac{1}{16 \pi G} \int d^{4} x \sqrt{-g}(R-2 \Lambda),
$$

where $G$ is the Newton constant, $R$ is the Ricci scalar and $\Lambda$ the cosmological constant. The Schwarzschild-dS and Schwarzschild-AdS black holes are described by the metric

$$
d s^{2}=f(r) d t^{2}-\frac{d r^{2}}{f(r)}-r^{2}\left(d \theta^{2}+\sin ^{2} \theta d \phi^{2}\right),
$$

where $f(r)=1-\frac{2 M}{r}-\frac{\Lambda r^{2}}{3}, M$ is the black hole mass, $\Lambda>0$ in the metric represents the Schwarzschild-dS black hole, while $\Lambda<0$ represents the Schwarzschild-AdS black hole. For 
the Schwarzschild-dS black hole the difference between the cosmological horizon $r_{\Lambda}$ and the event horizon $r_{H}$ decreases when the cosmological constant increases, and both horizons coincide when $9 M^{2} \Lambda=1$, while for the Schwarzschild-AdS black hole there is only one horizon that decreases when the absolute value of the cosmological constant increases.

The QNMs of scalar perturbations in the background of the metric (2.2) are given by the scalar field solution of the Klein-Gordon equation

$$
\frac{1}{\sqrt{-g}} \partial_{\mu}\left(\sqrt{-g} g^{\mu \nu} \partial_{\nu} \varphi\right)=-m^{2} \varphi
$$

with suitable boundary conditions for a black hole geometry. In the above expression $m$ is the mass of the scalar field $\varphi$. Now, by means of the following ansatz

$$
\varphi=e^{-i \omega t} R(r) Y(\Omega),
$$

the Klein-Gordon equation reduces to

$$
\frac{1}{r^{2}} \frac{d}{d r}\left(r^{2} f(r) \frac{d R}{d r}\right)+\left(\frac{\omega^{2}}{f(r)}+\frac{\kappa}{r^{2}}-m^{2}\right) R(r)=0
$$

where we defined $\kappa=-\ell(\ell+1)$, with $\ell=0,1,2, \ldots$, which represents the eigenvalue of the Laplacian on the two-sphere and $\ell$ is the multipole number. Now, defining $R(r)=\frac{F(r)}{r}$ and by using the tortoise coordinate $r^{*}$ given by $d r^{*}=\frac{d r}{f(r)}$, the Klein-Gordon equation can be written as a one-dimensional Schrödinger-like equation

$$
\frac{d^{2} F\left(r^{*}\right)}{d r^{* 2}}-V_{\mathrm{eff}}(r) F\left(r^{*}\right)=-\omega^{2} F\left(r^{*}\right),
$$

with an effective potential $V_{\text {eff }}(r)$, which parametrically thought, $V_{\text {eff }}\left(r^{*}\right)$, is given by

$$
V_{\text {eff }}(r)=-\frac{f(r)}{r^{2}}\left(\kappa-m^{2} r^{2}-f^{\prime}(r) r\right) .
$$

\subsection{Numerical analysis. Schwarzchild-de Sitter black holes}

Now, in order to compute the QNFs, we will solve numerically the differential equation (2.5) by using the pseudospectral Chebyshev method, see for instance [26]. First, it is convenient to perform a change of variable in order to limit the values of the radial coordinate to the range $[0,1]$. Thus, we define the change of variable $y=\left(r-r_{H}\right) /\left(r_{\Lambda}-r_{H}\right)$. So, the event horizon is located at $y=0$ and the cosmological horizon at $y=1$. The radial equation (2.5) becomes

$$
\begin{aligned}
& f(y) R^{\prime \prime}(y)+\left(\frac{2\left(r_{\Lambda}-r_{H}\right) f(y)}{r_{H}+\left(r_{\Lambda}-r_{H}\right) y}+f^{\prime}(y)\right) R^{\prime}(y) \\
& \quad+\left(r_{\Lambda}-r_{H}\right)^{2}\left(\frac{\omega^{2}}{f(y)}-\frac{\ell(\ell+1)}{\left(r_{H}+\left(r_{\Lambda}-r_{H}\right) y\right)^{2}}-m^{2}\right) R(y)=0 .
\end{aligned}
$$

In the vicinity of the horizon $(\mathrm{y} \rightarrow 0)$ the function $R(y)$ behaves as

$$
R(y)=C_{1} e^{-\frac{i \omega\left(r_{\Lambda}-r_{H}\right)}{f^{\prime}(0)} \ln y}+C_{2} e^{\frac{i \omega\left(r_{\Lambda}-r_{H}\right)}{f^{\prime}(0)} \ln y} .
$$


Here, the first term represents an ingoing wave and the second represents an outgoing wave near the black hole horizon. So, imposing the requirement of only ingoing waves at the horizon, we fix $C_{2}=0$. On the other hand, at the cosmological horizon the function $R(y)$ behaves as

$$
R(y)=D_{1} e^{-\frac{i \omega\left(r_{\Lambda}-r_{H}\right)}{f^{\prime}(1)} \ln (1-y)}+D_{2} e^{\frac{i \omega\left(r_{\Lambda}-r_{H}\right)}{f^{\prime}(1)} \ln (1-y)} .
$$

Here, the first term represents an outgoing wave and the second represents an ingoing wave near the cosmological horizon. So, imposing the requirement of only ingoing waves on the cosmological horizon requires $D_{1}=0$. Taking the behaviour of the scalar field at the event and cosmological horizons we define the following ansatz

$$
R(y)=e^{-\frac{i \omega\left(r_{\Lambda}-r_{H}\right)}{f^{\prime}(0)} \ln y} e^{\frac{i \omega\left(r_{\Lambda}-r_{H}\right)}{f^{\prime}(1)} \ln (1-y)} F(y) .
$$

Then, by inserting the above ansatz for $R(y)$ in eq. (2.8), an differential equation for the function $F(y)$ is obtained. The solution for the function $F(y)$ is assumed to be a finite linear combination of the Chebyshev polynomials, and it is inserted into the differential equation for $F(y)$. Also, the interval $[0,1]$ is discretized at the Chebyshev collocation points. Then, the differential equation is evaluated at each collocation point. So, a system of algebraic equations is obtained, and it corresponds to a generalized eigenvalue problem, which is solved numerically to obtain the QNFs $(\omega)$. In appendix A we show the accuracy of the numerical technique used.

Now, in order to visualize the different families of QNMs we plot in figure 1 the behaviour of $-\operatorname{Im}(\omega) M$ (left panel), and $\operatorname{Re}(\omega) M$ (right panel) as a function of $m M$ for different overtone numbers and $\ell=0$. In these figures we can recognize two families, for zero mass, a family of complex QNFs given by the black curves, and a purely imaginary family given by the blue dashed curves. The purely imaginary modes belong to the family of de Sitter modes, in that they continuously approach those of empty de Sitter in the limit that the black hole vanishes, while the complex ones are those of the Schwarzschild black hole, in the sense that they limit to the modes of the asymptotically at Schwarzschild black hole in that limit, and this family corresponds to the photon sphere modes. Also, we observe the behavior of the families when $m M$ the scalar field mass increases. We see that the photon sphere modes (black curves), is the dominant family for $m M>0.15$. Interestingly, the dS modes (colored curves) is the family dominant for $m M<0.15$; however, for some value of $m M$, the purely imaginary de Sitter modes (blue dashed curves) can acquire a real part, given by the continuous colored curves. In the next subsections, we study the QNFs for the photon sphere modes and for the dS modes separately.

\subsubsection{Photon sphere modes}

Now, in order to show the existence of anomalous decay rate of QNMs, we plot in figure 2 the behaviour of the fundamental QNFs of this family (top-left panel), for different values of the multipole number $\ell$, and different values of $m M$. The numerical values are in appendix B table 3. It is possible to observe that the imaginary part of these frequencies has an anomalous behaviour for $m M<m_{c} M$, i.e, the absolute value of the imaginary 

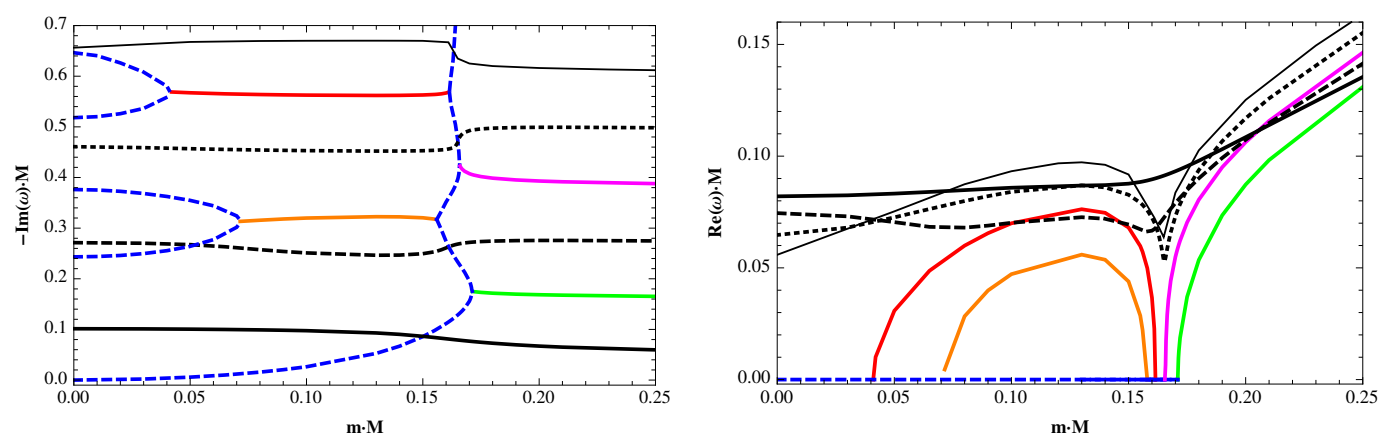

Figure 1. The behaviour of the QNFs, $-\operatorname{Im}(\omega) M$ (left panel), and $\operatorname{Re}(\omega) M$ (right panel), as a function of $m M$ for different overtone numbers, with $\ell=0$, and $M^{2} \Lambda=0.04$.

part decay when the angular harmonic numbers $\ell$ increase; however, for $m M>m_{c} M$ the behaviour is normal, i.e, the longest-lived modes are the ones with smaller angular number. The critical mass $m_{c}$ corresponds to the value of the scalar field mass where the behaviour in the QNFs is inverted, and can be obtained from the condition $\operatorname{Im}(\omega)_{\ell}=\operatorname{Im}(\omega)_{\ell+1}$ in the eikonal limit, that is when $\ell \rightarrow \infty$. Looking at figure 1 combined with figure 2 shows that the anomalous decay rate occurs at subdominant order for $m M<0.15$; however for $0.15<m M<m_{c} M$ the anomalous decay rate occurs at dominant order. Also, in order to show the same anomalous behaviour for the first overtone number $n_{P S}=1$, we plot in figure 2, the behaviour of $-\operatorname{Im}(\omega) M$ of the photon sphere modes (top-right panel), for $\ell \geq n_{P S}$ as a function of $m M .{ }^{1}$ Note that, the critical mass value increases when the overtone number $n_{P S}$ increases, for $\ell \geq n_{P S}$. The numerical values are in appendix $\mathrm{B}$ table 5. Also, the behaviour of the real part of the QNFs is smooth, and there is a slower decay of the mode when $m M$ increases, see appendix B table 3 and 5 . Also, it is observed that all the QNFs have negative imaginary part, which means that the propagation of scalar field is stable in this background. The bottom panel of figure 2 corresponds to a zoom of the top-left panel, we can appreciate better that for high values of $\ell$ the QNFs cross very close at the critical mass.

Now, in order to see the influence of the cosmological constant in the critical mass, we plot in figure 3 the behaviour of the fundamental QNFs, for different values of the multipole number $\ell$, and different values of the mass of the scalar field, but for a cosmological constant greater than the previous case $M^{2} \Lambda=0.11$. The numerical values are in appendix C table 6 . We can observe that for a greater cosmological constant the value of the critical mass increases, this will be confirmed analytically in the next section with the WKB formula.

It is interesting to note that, despite that the spacetime is asymptotically dS, where the boundary conditions are imposed at the event horizon and at the cosmological horizon

\footnotetext{
${ }^{1}$ We have left outside the case for $\ell=0$, because the imaginary part of the QNFs exhibits a behaviour other than $\ell>0$, that is, there is a region, where the absolute value of $-\operatorname{Im}(\omega) M$ increases when $m M$ increases, see figure 1 , and the curve for $\ell=0$ does not intersect the other curves, for the first overtone number $\left(n_{P S}=1\right)$. While that, for the dominant mode $\left(n_{P S}=0\right)$ the behaviour is opposite, and always is the same, that is, the absolute value of $-\operatorname{Im}(\omega) M$ always decreases, see figure 1 , and the curve for $\ell=0$ intersects the other curves. In general, this different behaviour occurs for $\ell<n_{P S}$.
} 

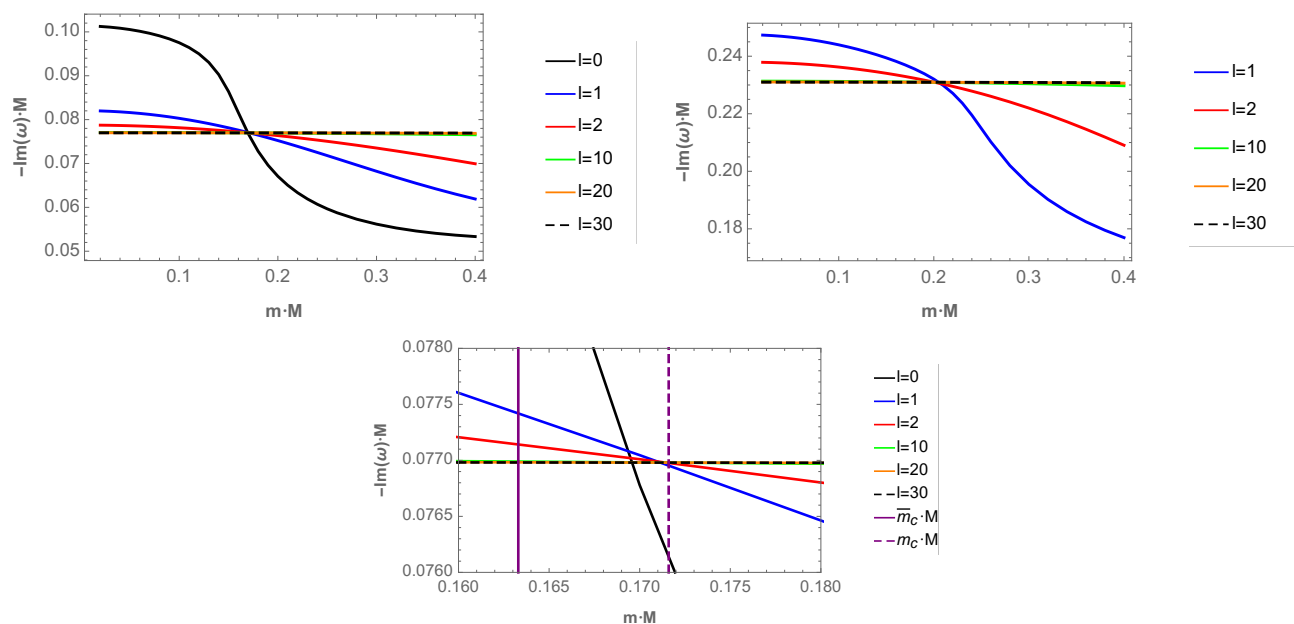

Figure 2. Top panel for the behaviour of $\operatorname{Im}(\omega) M$ of the photon sphere modes, top-left panel for the dominant mode $n_{P S}=0$, and right top-right panel for the first overtone $n_{P S}=1$, as a function of $m M$ for different values of the parameter $\ell=0,1,2,10,20,30$ (top-left panel), and $\ell=1,2,10,20,30 \geq n_{P S}$ (top-right panel), with $M^{2} \Lambda=0.04$. Bottom panel corresponds to a zoom of the top-left panel, for the range $0.16<m M<0.18, \bar{m}_{c} M \approx 0.1633$, and $m_{c} M \approx 0.1716$ (WKB).
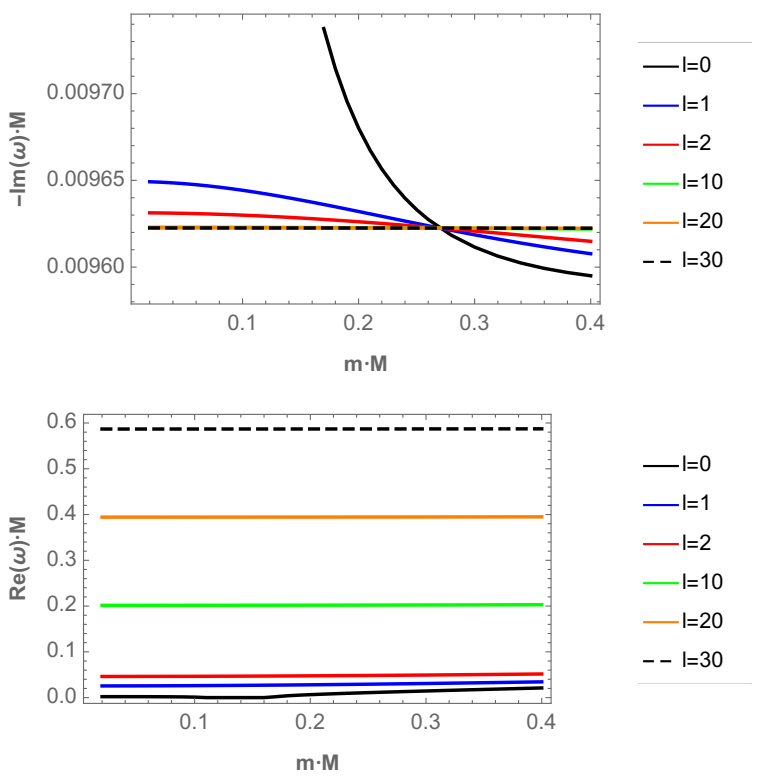

Figure 3. The behaviour of the QNFs for the dominant photon sphere modes $\left(n_{P S}=0\right), \operatorname{Im}(\omega) M$ (top panel) and $\operatorname{Re}(\omega) M$ (bottom panel), as a function of $m M$ for different values of the parameters $\ell=0,1,2,10,20,30$, with $M^{2} \Lambda=0.11$. 
the effective potential tends to $-\Lambda\left(3 m^{2}-2 \Lambda\right) r^{2} / 9$ for $\ell=0$, at infinity, and it can diverge positively, negatively, or be null, specifically, it vanishes for $m=\bar{m}_{c}= \pm \sqrt{2 \Lambda / 3}$. So, for $M^{2} \Lambda=0.04$, and for a scalar field with mass $m M=\bar{m}_{c} M \approx 0.163$, and also for $M^{2} \Lambda=0.11$ and for a scalar field with mass $m M=\bar{m}_{c} M \approx 0.278$, the effective potential vanishes at infinity. So, for a scalar field with $m=\bar{m}_{c}$, and $\ell=0$ the effective potential at infinity is not divergent. Also, for $\ell \neq 0$ and $m=\bar{m}_{c}$, the effective potential tends to a negative constant at infinity given by $-\ell(\ell+1) \Lambda / 3$ and the scalar field does not generate such divergence. It is worth mention this scalar field mass $\left(m=\bar{m}_{c}\right)$ also satisfies the lower limit on the value of the mass of the scalar field for the waves with QNFs related to the effective potential barrier between the black hole and the cosmological horizons of the Schwarzschild dS spacetime, and enabling the wave to reach observer at infinity without meeting any other potential wall proposed in ref. [39]. The physical picture behind it is that there is a specific critical scale of the scalar field that cancels out the scale introduced by the cosmological constant.

\subsection{2 dS modes}

These modes continuously approach those of empty de Sitter spacetime in the limit that the black hole vanishes, and the QNFs are given by [40]

$$
\omega=-i \sqrt{\frac{\Lambda}{3}}\left(\frac{3}{2}+\ell+2 n_{d S} \pm\left(\frac{9}{4}-\frac{3 m^{2}}{\Lambda}\right)^{1 / 2}\right),
$$

for $m^{2}<3 \Lambda / 4$ and

$$
\omega= \pm \sqrt{\frac{\Lambda}{3}}\left(-\frac{9}{4}+\frac{3 m^{2}}{\Lambda}\right)^{1 / 2}-i \sqrt{\frac{\Lambda}{3}}\left(\frac{3}{2}+\ell+2 n_{d S}\right)
$$

for $m^{2}>3 \Lambda / 4$, where $n_{d S}=0,1,2, \ldots$ Note that, for massless scalar field the QNFs are purely imaginary; however, for $m^{2}>3 \Lambda / 4$, the QNFs acquire a real part. The behaviour of the fundamental dS modes is showed in figure 4 as a function of $m M$ for different values of the multipole number and $M^{2} \Lambda=0.04$. We can observe that the modes of this family can acquire a real part depending on the scalar field mass, see table 4 . This happens approximately for $m M \approx 0.17$ in figure 4 . Thus, as it was observed all the QNFs are negative, which means that the propagation of scalar field is stable in this background. Moreover, we can observe a faster decay when the parameter $\ell$ increases, and a faster decay when the scalar field mass increases until the QNFs acquire a real part after which the decay is stabilized. Also, the real part increases when the scalar field mass increases. Thus, for the range of mass considered, the longest-lived modes are always the ones with lowest angular number, and the anomalous behaviour is not observed for this family of modes. 

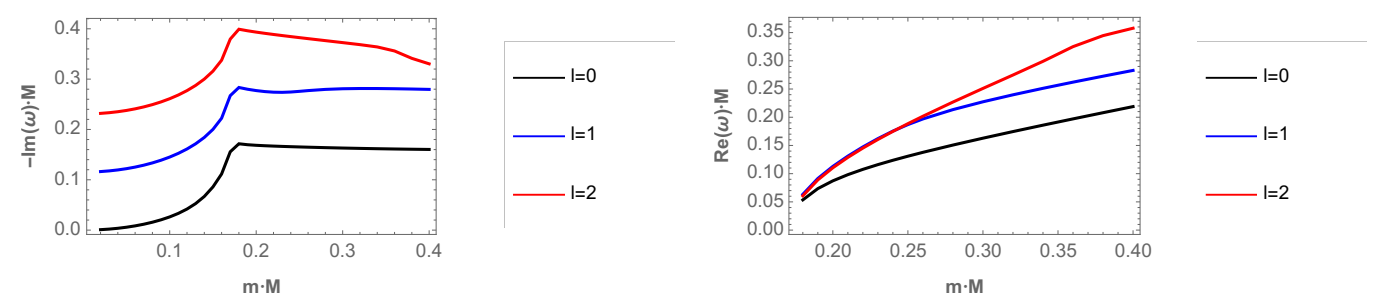

Figure 4. The behaviour of the dominant $\mathrm{dS}$ modes $\left(n_{d S}=0\right),-\operatorname{Im}(\omega) M$ (left pannel) and $\operatorname{Re}(\omega) M$ (right pannel) as a function of $m M$ for different values of the parameter $\ell=0,1,2$, with $M^{2} \Lambda=0.04$.

\subsection{Numerical analysis. Schwarzchild-AdS black holes}

In this case, under the change of variable $y=1-r_{H} / r$ the radial equation (2.5) becomes

$$
(1-y)^{4} f(y) R^{\prime \prime}(y)+(1-y)^{4} f^{\prime}(y) R^{\prime}(y)+\left(\frac{\omega^{2} r_{H}^{2}}{f(y)}-\ell(\ell+1)(1-y)^{2}-m^{2} r_{H}^{2}\right) R(y)=0,
$$

where the prime denotes derivative with respect to $y$. In the new coordinate the event horizon is located at $y=0$ and the spatial infinity at $y=1$. In the neighborhood of the horizon $(\mathrm{y} \rightarrow 0$ ) the function $R(y)$ behaves as

$$
R(y)=C_{1} e^{-\frac{i \omega r_{H}}{f^{\prime}(0)} \ln y}+C_{2} e^{\frac{i \omega r_{H}}{f^{\prime}(0)} \ln y},
$$

where the first term represents an ingoing wave and the second represents an outgoing wave near the black hole horizon. Imposing the requirement of only ingoing waves on the horizon, we fix $C_{2}=0$. Also, at infinity the function $R(y)$ behaves as

$$
R(y)=D_{1}(1-y)^{\frac{3}{2}+\sqrt{\left(\frac{3}{2}\right)^{2}-\frac{3 m^{2}}{\Lambda}}}+D_{2}(1-y)^{\frac{3}{2}-\sqrt{\left(\frac{3}{2}\right)^{2}-\frac{3 m^{2}}{\Lambda}}} .
$$

So, imposing the scalar field vanishes at infinity requires $D_{2}=0$. Therefore, by considering the behaviour at the event horizon and at infinity of the scalar field, it is possible to define the following ansatz

$$
R(y)=e^{-\frac{i \omega r_{H}}{f^{\prime}(0)} \ln y}(1-y)^{\frac{3}{2}+\sqrt{\left(\frac{3}{2}\right)^{2}-\frac{3 m^{2}}{\Lambda}}} F(y) .
$$

Then, by inserting this last expression in eq. (2.14) we obtain an equation for the function $F(y)$, which we solve numerically employing the pseudospectral Chebyshev method, as in the previous case. Also, in order to see if there is an anomalous decay rate of quasinormal modes, we plot in figure 5 the behaviour of the fundamental QNFs, for different values of the parameter $\ell$, and different values of $m M$. The numerical values are in appendix $\mathrm{D}$ table 7. We can observe an anomalous behaviour of the QNMs in Schwarzschild-AdS black holes because the longest-lived modes are always the ones with higher angular number; however, there is not a critical mass where the behaviour of the modes is inverted. The same behaviour is observed for $M^{2} \Lambda=-1$, see appendix D table 8. Also, they present a 

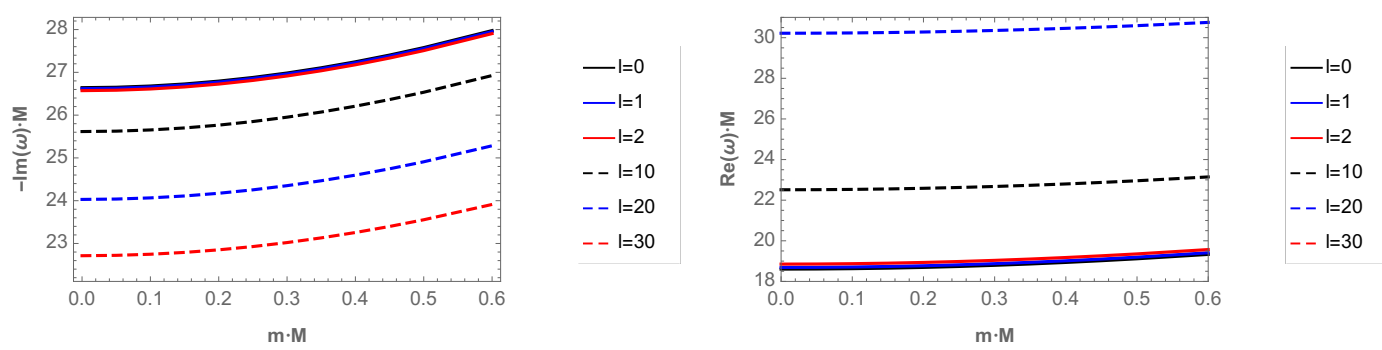

Figure 5. The behaviour of fundamental mode $-\operatorname{Im}(\omega) M$ (left figure) and $\operatorname{Re}(\omega) M$ (right figure) as a function of $m M$ for different values of the parameter $\ell=0,1,2,10,20,30$, with $M^{2} \Lambda=-765075$.
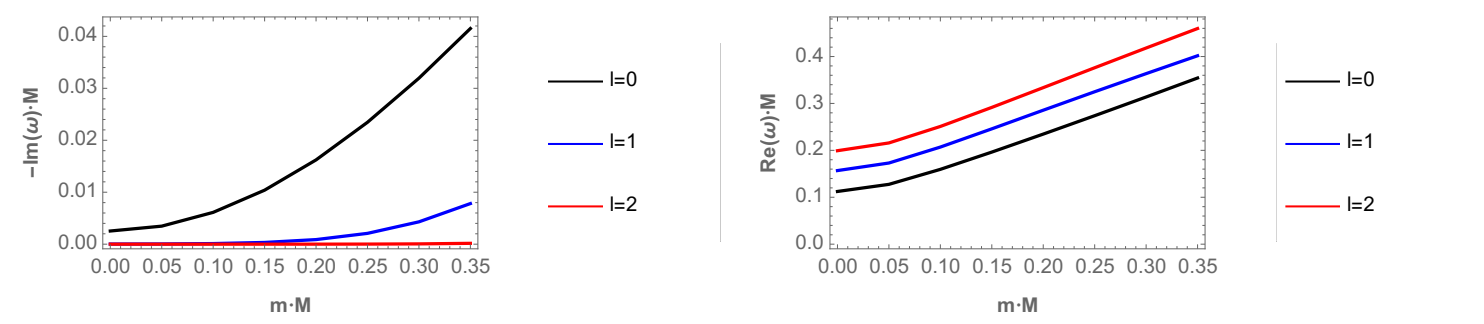

Figure 6. The behaviour of fundamental mode $-\operatorname{Im}(\omega) M$ (left figure) and $\operatorname{Re}(\omega) M$ (right figure) as a function of $m M$ for different values of the parameter $\ell=0,1,2$, with $M^{2} \Lambda=-0.005$.

faster decay when the scalar field mass increases and when the angular harmonic numbers $\ell$ decrease. The frequency of the oscillations increases sightly when the scalar field mass increases and also when the angular harmonic numbers $\ell$ decrease. On the other hand, the same behaviour is observed for small values of $M^{2} \Lambda$, and small $\ell$, see figure 6 , the numerical values are in appendix $\mathrm{D}$ table 9, where we found some QNFs for low values of $\ell=0,1,2$, however, for high values of $\ell$ the numerical method is not efficient, requiring a very large number of Chebyshev polynomials to converge due to the imaginary part becomes very small, which corresponds to weakly damped modes predicted by [41, 42], and analyzed further in [43]. It is worth mentioning that despite the analysis with respect to the critical mass was performed in the eikonal limit, even for small values of $\ell$ for the Schwarzschild dS case there is a reversal of the behaviour of the decay rate of the QNFs at a value of the mass very close to the critical mass of the scalar field, figure 2. We have not observed an reversal of the behaviour of the decay rate of the QNFs for Schwarzschild AdS black hole for small values of $\ell$, see figure 6 , and therefore we would not expect an inverted behaviour for high values of $\ell$ at some critical mass. However, it would be worthwhile to check this claim with some numerical method that works well in this limit, such as the method used in ref. [43].

In this case, the effective potential at infinity always diverges, due to the fact that the cosmological constant is negative, and consequently the scalar field can probe the divergence of the effective potential at infinity. 


\section{Analysis using the WKB method}

In this section, in order to get some analytical insight of the behavior of the QNFs in the eikonal limit $\ell \rightarrow \infty$, we use the method based on Wentzel-Kramers-Brillouin (WKB) approximation initiated by Mashhoon [44] and by Schutz and Iyer [45]. Iyer and Will computed the third order correction [46], and then it was extended to the sixth order [47], and recently up to the 13 th order [48], see also [49].

This method has been used to determine the QNFs for asymptotically flat and asymptotically de Sitter black holes. This is due to the WKB method can be used for effective potentials which have the form of potential barriers that approach to a constant value at the horizon and spatial infinity [5], so, it does apply to determine the QNFs of Schwarzschild (de-Sitter) black hole; however only the photon sphere modes can be obtained with this method. The QNMs are determined by the behavior of the effective potential near its maximum value $r_{\text {max }}^{*}$. The Taylor series expansion of the potential around its maximum is given by

$$
V\left(r^{*}\right)=V\left(r_{\max }^{*}\right)+\sum_{i=2}^{i=\infty} \frac{V^{(i)}}{i !}\left(r^{*}-r_{\max }^{*}\right)^{i},
$$

where

$$
V^{(i)}=\left.\frac{d^{i}}{d r^{* i}} V\left(r^{*}\right)\right|_{r^{*}=r_{\max }^{*}},
$$

corresponds to the $i$-th derivative of the potential with respect to $r^{*}$ evaluated at the maximum of the potential $r_{\max }^{*}$. Using the WKB approximation up to 6th order the QNFs are given by the following expression [50]

$$
\omega^{2}=V\left(r_{\max }^{*}\right)-2 i U,
$$

where

$$
\begin{aligned}
U= & N \sqrt{-V^{(2)} / 2}+\frac{i}{64}\left(-\frac{1}{9} \frac{V^{(3) 2}}{V^{(2) 2}}\left(7+60 N^{2}\right)+\frac{V^{(4)}}{V^{(2)}}\left(1+4 N^{2}\right)\right) \\
& +\frac{N}{2^{3 / 2} 288}\left(\frac{5}{24} \frac{V^{(3) 4}}{\left(-V^{(2)}\right)^{9 / 2}}\left(77+188 N^{2}\right)+\frac{3}{4} \frac{V^{(3) 2} V^{(4)}}{\left(-V^{(2)}\right)^{7 / 2}}\left(51+100 N^{2}\right)\right. \\
& \left.+\frac{1}{8} \frac{V^{(4) 2}}{\left(-V^{(2)}\right)^{5 / 2}}\left(67+68 N^{2}\right)+\frac{V^{(3)} V^{(5)}}{\left(-V^{(2)}\right)^{5 / 2}}\left(19+28 N^{2}\right)+\frac{V^{(6)}}{\left(-V^{(2)}\right)^{3 / 2}}\left(5+4 N^{2}\right)\right),
\end{aligned}
$$

and $N=n_{P S}+1 / 2$, with $n_{P S}=0,1,2, \ldots$, is the overtone number. The imaginary and real part of the QNFs can be written as

$$
\begin{aligned}
\omega_{I}^{2} & =-(\operatorname{Im}(U)+V / 2)+\sqrt{(\operatorname{Im}(U)+V / 2)^{2}+\operatorname{Re}(U)^{2}}, \\
\omega_{R}^{2} & =-\operatorname{Re}(U)^{2} / \omega_{I}^{2},
\end{aligned}
$$

respectively, where $\operatorname{Re}(U)$ is the real part of $U$ and $\operatorname{Im}(U)$ its imaginary part. 
Defining $L^{2}=\ell(\ell+1)$, we find that for large values of $L$, the maximum of the potential is approximately at

$$
r_{\max } \approx 3 M-\frac{M}{3 L^{2}}\left(1-27 m^{2} M^{2}+18 \Lambda M^{2}\right) \mathcal{B}
$$

and

$$
V\left(r_{\max }^{*}\right) \approx \mathcal{B}\left(\frac{L^{2}}{27 M^{2}}+\frac{2+27 m^{2} M^{2}-18 \Lambda M^{2}}{81 M^{2}}\right)
$$

where

$$
\mathcal{B}=1-9 \Lambda M^{2},
$$

while the second derivative of the potential evaluated at $r_{\max }^{*}$ yields

$$
V^{(2)}\left(r_{\max }^{*}\right) \approx \mathcal{B}^{2}\left(-\frac{2 L^{2}}{729 M^{4}}-\frac{2 \mathcal{A}}{6561 M^{4}}\right)
$$

where

$$
\mathcal{A}=8-54 m^{2} M^{2}+18 \Lambda M^{2}+1215 m^{2} M^{4} \Lambda-810 \Lambda^{2} M^{4} .
$$

For the higher derivatives, only the leading terms are important in the limit considered

$$
\begin{aligned}
V^{(3)}\left(r_{\max }^{*}\right) & \approx \mathcal{B}^{3} \frac{4 L^{2}}{6561 M^{5}}, \\
V^{(4)}\left(r_{\max }^{*}\right) & \approx \mathcal{B}^{3} \frac{16 L^{2}}{19683 M^{6}}, \\
V^{(5)}\left(r_{\max }^{*}\right) & \approx-\mathcal{B}^{4} \frac{40 L^{2}}{59049 M^{7}}, \\
V^{(6)}\left(r_{\max }^{*}\right) & \approx-\mathcal{B}^{4} \frac{16 L^{2}\left(4+15 \Lambda M^{2}\right)}{177147 M^{8}} .
\end{aligned}
$$

Using these results we find that $U$ evaluated at $r_{\max }^{*}$ is given approximately by

$$
\begin{aligned}
U \approx \mathcal{B} & {\left[\frac{\left(1+2 n_{P S}\right) L}{54 M^{2}}+i \frac{-65+99 \Lambda M^{2}+6 n_{P S}\left(n_{P S}+1\right)\left(-23+45 \Lambda M^{2}\right)}{5832 M^{2}}+\frac{1+2 n_{P S}}{209952 M^{2} L}(749\right.} \\
& -5 n_{P S}\left(n_{P S}+1\right) \mathcal{B}\left(61+423 \Lambda M^{2}\right)-11664 m^{2} M^{2}+262440 m^{2} M^{4} \Lambda+6930 \Lambda M^{2} \\
& \left.\left.-162405 \Lambda^{2} M^{4}\right)-i \frac{\left(-38+99 \Lambda M^{2}+6 n_{P S}\left(n_{P S}+1\right)\left(-14+45 \Lambda M^{2}\right)\right) \mathcal{A}}{26244 M^{2} L^{2}}\right],
\end{aligned}
$$

thereby, by using eq. (3.4) we obtain

$$
\begin{aligned}
\omega_{I}^{2} \approx & \frac{\left(1+2 n_{P S}\right)^{2} \mathcal{B}}{M^{2}}\left(\frac{1}{108}\right. \\
& \left.+\frac{\mathcal{B}\left(137-29160 m^{2} M^{2}+18045 \Lambda M^{2}+235 n_{P S}\left(n_{P S}+1\right) \mathcal{B}\right)}{209952 L^{2}}\right)
\end{aligned}
$$

The second term of this expression is zero at the value of the critical mass $m_{c}$, which is given by

$$
m_{c} M=\sqrt{\frac{137+18045 \Lambda M^{2}+235 n_{P S}\left(n_{P S}+1\right)\left(1-9 \Lambda M^{2}\right)}{29160}} .
$$




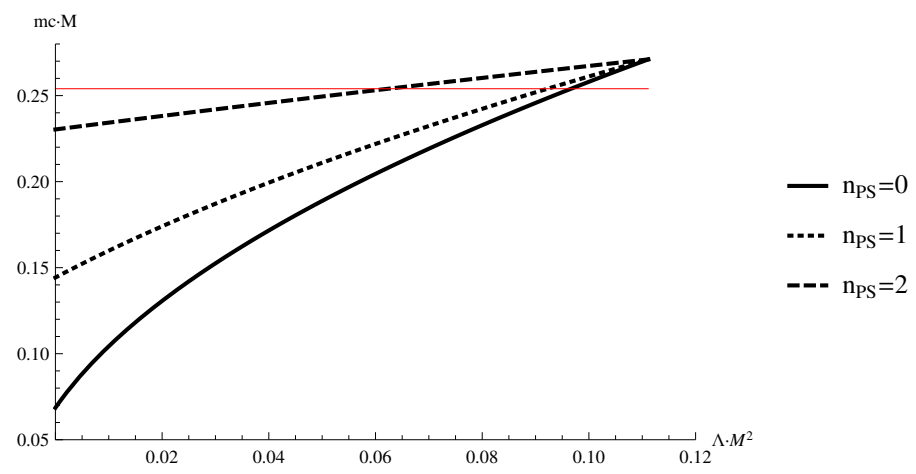

Figure 7. The behaviour of $m_{c} M$ as a function of $\Lambda M^{2}$ for different values of the overtone number $n_{P S}=0,1$, and 2 . For values of the critical mass below the horizontal red line the zero mode is subdominant at the critical mass, while it is dominant at the critical mass for values above the line.

Therefore, we can observe an inverted behavior of $\omega_{I}(\ell)$. For $m>m_{c}, \omega_{I}$ increases with $\ell$; whereas, for $m<m_{c}, \omega_{I}$ decreases when $\ell$ increases.

For $\Lambda=0$ we recover the eq. (28) of ref. [14]. For $\Lambda M^{2}=0.04$ and $n_{P S}=0$ we obtain from (3.17) the value $m_{c} M=0.1716$ and for $n_{P S}=1$ we obtain $m_{c} M=0.1994$, which agrees with the numerical results shown in figure 2 for high values of $\ell$. For $\Lambda M^{2}=0.11$ and $n_{P S}=0$ we obtain $m_{c} M=0.2698$ which agrees with the numerical result shown in figure 3. In figure 7, we plot the behaviour of $m_{c} M$ as a function of $M^{2} \Lambda$ for different values of the overtone number. We can see that, for small values of $M^{2} \Lambda$ the critical mass depends on the overtone number $n_{P S}$, that is, the critical mass value increases when the overtone number increases. However, when $M^{2} \Lambda$ increases, the black hole becomes extremal when $9 M^{2} \Lambda=1$, and the critical mass value does not depend on the overtone number, the same behaviour was observed in the context of $f(R)$ gravity [51]. We have found numerically, using the pseudospectral method, that the $\ell=0$ mode that goes to a zero mode in the zero mass limit is subdominant for critical mass $m_{c} M<m_{*} M$, while it is dominant for $m_{c} M>m_{*} M$, where $m_{*} M \approx 0.2544$ (which corresponds to $\Lambda M^{2} \approx 0.097$ and $n_{P S}=0$ ). The horizontal red line of figure 7 corresponds to $m_{c} M=m_{*} M$, therefore, for values of critical mass below the line, the zero mode is subdominant at the critical mass, while it is dominant at the critical mass for values above the line. Note that naively extrapolating the WKB result eq. (3.17) for the critical mass to negative $\Lambda,{ }^{2}$ it is possible to obtain a positive critical mass for $\Lambda M^{2}>-0.007$ and $n_{P S}=0$, which suggests that could exist a range of small AdS curvature with a nonzero critical mass. Thus, for $\Lambda M^{2}=-0.005$, and $n_{P S}=0, \mathrm{WKB}$ analysis could predict a critical mass at $m_{c} M \approx 0.04$. However, as we mentioned in the previous subsection, we did not find any indication of a critical mass for Schwarzschild AdS black hole.

Now, we perform a analysis similar to ref. [14] to show an inverted behavior of the width of the potential in the case of Schwarzschild de-Sitter black hole. The coefficients $V^{(i)}$ of the Taylor expansion of the potential around its maximum in the large $\ell$ expansion

\footnotetext{
${ }^{2}$ We thank the referee for pointing out this fact to us.
} 
can be written as

$$
V^{(i)}=A^{(i)} L^{2}+B^{(i)}
$$

where the leading coefficients $A^{(i)}$ are given in eqs. (3.7)-(3.14). Thus, we obtain

$$
\omega_{I}^{2}=\frac{\mathcal{B}}{108 M^{2}}-\frac{5 \mathcal{B}^{3}\left(59+279 \Lambda M^{2}\right)+52488 M^{2} \mathcal{B} B^{(0)}+708588 M^{4} B^{(2)}}{209952 M^{2} \mathcal{B} L^{2}} .
$$

This eq. can be expressed as

$$
\omega_{I}^{2}=-\frac{1}{8} \frac{V^{(2)}}{V\left(r_{\max }^{*}\right)}-\frac{5 \mathcal{B}^{2}\left(59+279 \Lambda M^{2}\right)}{209952 M^{2} L^{2}} .
$$

In this expression $V$ and $V^{(2)}$ consider their leading and sub-leading order terms when $\ell \rightarrow \infty$. The first term of this expression can be written in terms of the maximum height of the potential and its width around $r_{\max }^{*}$. This can be seen expanding the potential around $r_{\max }^{*}$ up to second order derivatives:

$$
V\left(r^{*}\right) \approx V\left(r_{\max }^{*}\right)+\frac{V^{(2)}}{2}\left(r^{*}-r_{\max }^{*}\right)^{2}
$$

From here, considering an $r^{*}$ near $r_{\max }^{*}$ such that $V\left(r^{*}\right)=\epsilon V\left(r_{\max }^{*}\right)$, and defining $\Delta r^{*}=$ $r^{*}-r_{\max }^{*}$, we obtain $\left(\Delta r^{*}\right)^{2} \approx-2(1-\epsilon) V\left(r_{\max }^{*}\right) / V^{(2)}$. Thus, the imaginary part of the QNFs to sub-leading order are given by

$$
\omega_{I}^{2}=\frac{(1-\epsilon)}{4\left(\Delta r^{*}\right)^{2}}-\frac{5 \mathcal{B}^{2}\left(59+279 \Lambda M^{2}\right)}{209952 M^{2} L^{2}} .
$$

Therefore, the width is found to behaves, to second order, as

$$
\left(\Delta r^{*}\right)^{2} \approx \frac{3(1-\epsilon) M^{2}}{\mathcal{B}}\left(9+\frac{1}{L^{2}} \mathcal{B}\left(-2+135 m^{2} M^{2}-90 \Lambda M^{2}\right)\right),
$$

and exhibit an inverted behavior. For $\Lambda=0$ the result of [14] is recovered. We note that this inversion in the behaviour of the width does not happen exactly at the same critical value of the mass where imaginary part of the QNFs exhibits an inverted behaviour, as in ref. [14]. However, certainly it is responsible of the inverted behaviour of $\omega_{I}$.

\section{Conclusions}

In this work, we considered the Schwarzschild-dS and the Schwarzschild-AdS black hole as backgrounds and we studied the propagation of massive scalar fields through the QNFs by using the pseudospectral Chebyshev method in order to determine if there is an anomalous decay behaviour in the QNMs as it was observed in the asymptotically flat Schwarzschild black hole background.

The QNMs of a massless scalar field in the background of a Schwarzschild-dS black hole are characterized by two families of modes, the photon sphere modes which are complex and the dS modes consisting of purely imaginary QNFs. The dS modes are generated for 
small scalar field mass and eventually this family can acquires a real part when the mass of the scalar field increases, and it is worth to mention that to our knowledge, this is the first time that this behaviour has been reported. Both families present frequencies with a negative imaginary part, which means that the propagation of scalar field is stable in this background.

For the photon sphere modes, the presence of the cosmological constant leads to decrease the real oscillation frequency and to a slower decay [35]. We showed that for the dominant QNFs there is a slower decay rate when the mass of the scalar field increases for a fixed angular harmonic number $\ell$. Furthermore, we showed the existence of anomalous decay rate of QNMs, i.e, the absolute values of the imaginary part of the QNFs decay when the angular harmonic numbers increase if the mass of the scalar field is smaller than a critical mass. On the contrary they grow when the angular harmonic numbers increase, if the mass of the scalar field is larger than the critical mass and they also increase with the overtone number $n_{P S}$, for $\ell \geq n_{P S}$. We also showed that the effect of the cosmological constant is to shift the values of the critical mass i.e the value of the critical mass increases with the cosmological constant. Moreover, by using the WKB method, we found that the critical mass for large $\ell$ is given by $m_{c} M=\sqrt{\left(137+18045 \Lambda M^{2}\right) / 29160}$, for the dominant mode $n_{P S}=0$. It is worth to mention here that the critical mass is an interesting quantity, because it shows that it is possible to have a scalar field with a critical mass and the decay rate does not depend appreciably on the angular harmonic numbers $\ell$; however, its frequency of oscillation depends on the angular harmonic numbers $\ell$, increasing when $\ell$ increases.

For the dS modes, it was shown, depending on the black hole mass it may even be the dominant mode [37]. We found that for a fixed value of the black hole mass, the purely imaginary QNFs can also be dominant depending on the scalar field mass and the angular harmonic numbers. Additionally, a faster decay is observed when the $\ell$ parameter increases, as well as, when the scalar field mass increases until that the QNFs acquire a real part, after it the decay is stabilized, and the frequency of the oscillations increases when the scalar field mass increases. Furthermore, we showed that this family does not present an anomalous behaviour of the QNFs, for the range of scalar field mass analyzed.

In the case of a Schwarzschild-AdS black hole background we have shown that the QNMs of massive scalar fields under Dirichlet boundary conditions present an anomalous behaviour of the QNMS because the longest-lived modes are always the ones with higher angular number. Also, they present a faster decay when the scalar field mass increases. However, we showed that for large and intermediate black holes there is not a critical mass where the behaviour of the modes is inverted. For small black holes our analysis was performed only for low values of $\ell$, suggesting that neither there is a critical mass in this case. However, it would be worthwhile to check this claim with some numerical method that works well for high $\ell$.

Therefore, the anomalous behaviour in the QNFs is possible in asymptotically flat, in asymptotically $\mathrm{dS}$ and in asymptotically AdS spacetimes; however, we observed that the critical mass exist for asymptotically flat and for asymptotically dS spacetimes and it is not present in asymptotically AdS spacetimes for large and intermediate black holes. Also, 
as we mentioned, through our analysis of low values of $\ell$ there is not indication that it could be present for small asymptotically AdS black holes. The existence of the critical mass could depend if the scalar field probes the divergence of the effective potential at infinity, despite that the boundary conditions can be imposed in a different point. It is worth to mention that for a Schwarzschild black hole, the effective potential tends to $\mathrm{m}^{2}$, so the scalar field does not probe the divergence, and consequently a critical mass can be observed. Note that, for the geometries studied, the critical mass is only present for the photon sphere modes, which are well described by the WKB approximation, and they approach asymptotically to the QNMS of Schwarzschild black hole for very small value of the cosmological constant. On the other hand, the QNMs of Schwarzschild AdS black hole do not approach asymptotically to the QNMS of Schwarzschild black hole for very small cosmological constant, due to the boundary conditions at spatial infinity and the effective potentials are very different.

It would be interesting to extent this work to the case the background black hole is charged and study the behaviour of QNMs in this background and in different asymptotic spacetimes. If the background metric is the Reissner-Nordström black hole in dS spacetime an interesting effect in Gravity theory and in its scalar-tensor extension, is the Strong Cosmic Censorship (SCC) effect [38, 52]. It was found in [53] that there intervals of the masses and charges of the scalar field that we have violation or not of SCC. Therefore it would be interesting to study how this behaviour of SCC is connected with the anomalous decay of the QNMs and the critical mass of the scalar field beyond which these decays occur.

\section{A Accuracy of the numerical method}

In table 1 we show some dominant photon sphere modes, in order to check the correctness and accuracy of the numerical technique used. Also, we show the relative error, which is defined by

$$
\begin{aligned}
\epsilon_{R e(\omega)} & =\frac{\left|\operatorname{Re}\left(\omega_{1}\right)-\operatorname{Re}\left(\omega_{0}\right)\right|}{\operatorname{Re}\left(\omega_{0}\right)} \cdot 100 \% \\
\epsilon_{\operatorname{Im}(\omega)} & =\frac{\left|\operatorname{Im}\left(\omega_{1}\right)-\operatorname{Im}\left(\omega_{0}\right)\right|}{\operatorname{Im}\left(\omega_{0}\right)} \cdot 100 \%
\end{aligned}
$$

where $\omega_{1}$ corresponds to the result from [35], and $\omega_{0}$ denotes our result. The complex QNFs for this geometry was determined in ref. [35] by using the WKB and Pöschl-Teller method. We can observed that error does not exceed $0.37 \%$ when we compare our results with the WKB method and $2.198 \%$ with the P-T method. As it was observed, the frequencies all have a negative imaginary part, which means that the propagation of scalar field is stable in this background. Also, we observe that the presence of a bigger cosmological constant leads to decrease the real oscillation frequency and to a slower decay.

On the other hand, in [37] another branch of purely imaginary QNFs was found for this geometry by using the pseudospectral Chebyshev method, with the metric expressed in Eddington-Finkelstein coordinates. Here, we have considered the coordinates given by 


\begin{tabular}{|c|c|c|c|c|c|c|c|}
\hline$\Lambda$ & $\omega_{W K B}$ & $\omega_{P-T}$ & $\omega$ & $\epsilon_{\operatorname{Re}(\omega)}(W K B)$ & $\epsilon_{\operatorname{Im}(\omega)}(W K B)$ & $\epsilon_{\operatorname{Re}(\omega)}(P-T)$ & $\epsilon_{\operatorname{Im}(\omega)}(P-T)$ \\
\hline 0.02 & $0.2603-0.0911 i$ & $0.263-0.093 i$ & $0.2603-0.0910 i$ & 0.000 & 0.110 & 1.037 & 2.198 \\
0.04 & $0.2247-0.0821 i$ & $0.226-0.083 i$ & $0.2247-0.0821 i$ & 0.045 & 0.122 & 0.623 & 1.220 \\
0.06 & $0.1854-0.0701 i$ & $0.187-0.071 i$ & $0.1854-0.0701 i$ & 0.054 & 0.143 & 0.917 & 1.429 \\
0.08 & $0.1404-0.0542 i$ & $0.141-0.055 i$ & $0.1404-0.0540 i$ & 0.000 & 0.370 & 0.427 & 1.852 \\
0.09 & $0.11392-0.04397 i$ & $0.1147-0.0443 i$ & $0.11400-0.04388 i$ & 0.070 & 0.205 & 0.614 & 0.957 \\
0.10 & $0.08156-0.03121 i$ & $0.0819-0.0315 i$ & $0.08159-0.03123 i$ & 0.037 & 0.064 & 0.380 & 0.865 \\
0.11 & $0.02549-0.00965 i$ & $0.02550-0.00967 i$ & $0.02549-0.00965 i$ & 0.000 & 0.000 & 0.039 & 0.207 \\
\hline
\end{tabular}

Table 1. Dominant photon sphere modes $\left(n_{P S}=0\right)$ for massless scalar fields with $\ell=1$ in the background of Schwarzchild-de Sitter black holes. The values of $\omega_{W K B}$ and $\omega_{P-T}$ appear in ref. [35].

\begin{tabular}{|c|c|c|}
\hline$\Lambda$ & $\omega_{I}$ & $\omega$ \\
\hline 0.02 & $-0.081565496 i$ & $-0.081565496 i$ \\
0.04 & $-0.11524810 i$ & $-0.11524810 i$ \\
0.06 & $-0.14100253 i$ & $-0.14100253 i$ \\
0.08 & $-0.16268011 i$ & $-0.16268011 i$ \\
0.09 & $-0.17249210 i$ & $-0.17249210 i$ \\
0.10 & $-0.18177480 i$ & $-0.18177480 i$ \\
0.11 & $-0.19057630 i$ & $-0.19057630 i$ \\
\hline
\end{tabular}

Table 2. Dominant dS modes $\left(n_{d S}=0\right)$ for massless scalar fields with $\ell=1$ in the background of Schwarzchild-de Sitter black holes with $M=1$. The values of $\omega_{I}$ appear in ref. [37].

the metric. Eq. (2.2) along with the change of variables $y=\left(r-r_{H}\right) /\left(r_{\Lambda}-r_{H}\right)$. Now, in order to check the correctness and accuracy of the numerical techniques used, we show the dominant dS modes in table 2, where the relative error vanishes. As it was observed, the frequencies all are negative, which means that the propagation of scalar field is stable in this background. However, the presence of the cosmological constant leads to a fast decay, when it increases, that is, contrary to the complex QNFs. Also, it was shown that depending on the black hole mass may even be the dominant modes [37].

\section{B Numerical values. Schwarzschild-de Sitter black hole with $M^{2} \Lambda=0.04$}

In this appendix we provide the numerical values of the QNFs. All complex QNFs come with their negative complex conjugate which we do not show. In table 3 and 4 we show for both families the dominant modes for massive scalar fields in the background of a Schwarzschild-de Sitter black hole, and in table 5, the numerical values of the photon sphere mode for the first overtone number $\left(n_{P S}=1\right)$. 


\begin{tabular}{|c|c|c|c|c|}
\hline$\ell$ & $m M=0.02$ & $m M=0.03$ & $m M=0.04$ & $m M=0.05$ \\
\hline 0 & $0.08221623-0.10123911 i$ & $0.08246910-0.10107321 i$ & $0.08281206-0.10083840 i$ & $0.08323382-0.10053157 i$ \\
\hline 1 & $0.22485188-0.08198069 i$ & $0.22506071-0.08189246 i$ & $0.22535335-0.08176899 i$ & $0.22573006-0.08161032 i$ \\
\hline 2 & $0.38089209-0.07873423 i$ & $0.38102729-0.07870351 i$ & $0.38121659-0.07866053 i$ & $0.38145998-0.07860531 i$ \\
\hline 10 & $1.61559336-0.07707753 i$ & $1.61562631-0.07707585 i$ & $1.61567245-0.07707350 i$ & $1.61573177-0.07707047 i$ \\
\hline 20 & $3.15567507-0.07700559 i$ & $3.15569196-0.07700515 i$ & $3.15571561-0.07700453 i$ & $3.15574602-0.07700374 i$ \\
\hline 30 & $4.69544173-0.07699158 i$ & $4.69545309-0.07699138 i$ & $4.69546899-0.07699110 i$ & $4.69548943-0.07699074 i$ \\
\hline$\ell$ & $m M=0.06$ & $m M=0.07$ & $m M=0.08$ & $m M=0.09$ \\
\hline 0 & $0.08371955-0.10014667 i$ & $0.08425049-0.09967430 i$ & $0.08480354-0.09909918 i$ & $0.08535077-0.09839688 i$ \\
\hline 1 & $0.22619110-0.08141653 i$ & $0.22673721-0.08118773 i$ & $0.22736862-0.08092405 i$ & $0.22808606-0.08062571 i$ \\
\hline 2 & $0.38175751-0.07853780 i$ & $0.38210917-0.07845832 i$ & $0.38251490-0.07836665 i$ & $0.38297502-0.07826293 i$ \\
\hline 10 & $1.61580427-0.07706678 i$ & $1.61588995-0.07706241 i$ & $1.61598882-0.07705737 i$ & $1.61610086-0.07705166 i$ \\
\hline 20 & $3.15578319-0.07700277 i$ & $3.15582711-0.07700163 i$ & $3.15587779-0.07700031 i$ & $3.15593522-0.07699881 i$ \\
\hline 30 & $4.69551441-0.07699031 i$ & $4.69554394-0.07698979 i$ & $4.69557801-0.07698919 i$ & $4.69561662-0.07698852 i$ \\
\hline$\ell$ & $m M=0.10$ & $m M=0.11$ & $m M=0.12$ & $m M=0.13$ \\
\hline 0 & $0.08585920-0.09752810 i$ & $0.08629220-0.09642921 i$ & $0.08661627-0.09499337 i$ & $0.08682704-0.09304079 i$ \\
\hline 1 & $0.22889025-0.08029297 i$ & $0.22978202-0.07992618 i$ & $0.23076229-0.07952580 i$ & $0.23183206-0.07909243 i$ \\
\hline 2 & $0.38348926-0.07814724 i$ & $0.38405777-0.07801965 i$ & $0.38468056-0.07788026 i$ & $0.38535769-0.07772916 i$ \\
\hline 10 & $1.61622608-0.07704529 i$ & $1.61636449-0.07703824 i$ & $1.61651607-0.07703052 i$ & $1.61668084-0.07702213 i i$ \\
\hline 20 & $3.15599942-0.07699714 i$ & $3.15607037-0.07699530 i$ & $3.15614808-0.07699327 i$ & $3.15623254-0.07699107 i$ \\
\hline 30 & $4.69565977-0.07698776 i$ & $4.69570747-0.07698693 i$ & $4.69575971-0.07698601 i$ & $4.69581649-0.07698502 i$ \\
\hline$\ell$ & $m M=0.14$ & $m M=0.15$ & $m M=0.16$ & $m M=0.17$ \\
\hline 0 & $0.08703483-0.09028329 i$ & $0.08767928-0.08640066 i$ & $0.08961851-0.08156211 i$ & $0.09323258-0.07677868 i$ \\
\hline 1 & $0.23299242-0.07862680 i$ & $0.23424454-0.07812982 i$ & $0.23558962-0.07760263 i$ & $0.23702888-0.07704656 i$ \\
\hline 2 & $0.38608920-0.07756646 i$ & $0.38687512-0.07739228 i$ & $0.38771552-0.07720675 i$ & $0.38861043-0.07701000 i$ \\
\hline 10 & $1.61685878-0.07701307 i$ & $1.61704990-0.07700334 i$ & $1.61725420-0.07699294 i$ & $1.61747167-0.07698188 i$ \\
\hline 20 & $3.15632376-0.07698870 i$ & $3.15642174-0.07698615 i$ & $3.15652647-0.07698342 i$ & $3.15663796-0.07698052 i$ \\
\hline 30 & $4.69587781-0.07698395 i$ & $4.69594368-0.07698280 i$ & $4.69601408-0.07698157 i$ & $4.69608903-0.07698025 i$ \\
\hline$\ell$ & $m M=0.18$ & $m M=0.19$ & $m M=0.20$ & $m M=0.21$ \\
\hline 0 & $0.09793849-0.07278715 i$ & $0.10312414-0.06961851 i$ & $0.10848564-0.06709019 i$ & $0.11389788-0.06503633 i$ \\
\hline 1 & $0.23856353-0.07646322 i$ & $0.24019471-0.07585443 i$ & $0.24192347-0.07522232 i$ & $0.24375068-0.07456924 i$ \\
\hline 2 & $0.38955991-0.07680221 i$ & $0.39056402-0.07658352 i$ & $0.39162279-0.07635413 i$ & $0.39273630-0.07611422 i$ \\
\hline 10 & $1.61770232-0.07697014 i$ & $1.61794614-0.07695775 i$ & $1.61820314-0.07694468 i$ & $1.61847332-0.07693095 i$ \\
\hline 20 & $3.15675621-0.07697744 i$ & $3.15688122-0.07697419 i$ & $3.15701298-0.07697076 i$ & $3.15715149-0.07696716 i$ \\
\hline 30 & $4.69616853-0.07697886 i$ & $4.69625256-0.07697739 i$ & $4.69634114-0.07697585 i$ & $4.69643426-0.07697422 i$ \\
\hline$\ell$ & $m M=0.22$ & $m M=0.23$ & $m M=0.24$ & $m M=0.25$ \\
\hline 0 & $0.11931155-0.06333786 i$ & $0.12470831-0.06191201 i$ & $0.13008281-0.06070044 i$ & $0.13543506-0.05966101 i$ \\
\hline 1 & $0.24567703-0.07389780 i$ & $0.24770294-0.07321081 i$ & $0.24982855-0.07251125 i$ & $0.25205365-0.07180223 i$ \\
\hline 2 & $0.39390459-0.07586401 i$ & $0.39512771-0.07560372 i$ & $0.39640571-0.07533360 i$ & $0.39773863-0.07505389 i$ \\
\hline 10 & $1.61875666-0.07691655 i$ & $1.61905318-0.07690149 i$ & $1.61936287-0.07688577 i$ & $1.61968573-0.07686938 i$ \\
\hline 20 & $3.15729676-0.07696338 i$ & $3.15744879-0.07695942 i$ & $3.15760757-0.07695529 i$ & $3.15777311-0.07695099 i$ \\
\hline 30 & $4.69653192-0.07697251 i$ & $4.69663412-0.07697072 i$ & $4.69674086-0.07696886 i$ & $4.69685215-0.07696691 i$ \\
\hline$\ell$ & $m M=0.26$ & $m M=0.28$ & $m M=0.30$ & $m M=0.32$ \\
\hline 0 & $0.14076723-0.05876242 i$ & $0.15138316-0.05729793 i$ & $0.16195355-0.05617189 i$ & $0.17249742-0.05529719 i$ \\
\hline 1 & $0.25437769-0.07108691 i$ & $0.25931866-0.06965002 i$ & $0.26464006-0.06822498 i$ & $0.27032567-0.06683365 i$ \\
\hline 2 & $0.39912653-0.07476488 i$ & $0.40206736-0.07416012 i$ & $0.40522840-0.07352189 i$ & $0.40860970-0.07285303 i$ \\
\hline 10 & $1.62002176-0.07685233 i$ & $1.62073331-0.07681625 i$ & $1.62149753-0.07677753 i$ & $1.62231440-0.07673618 i$ \\
\hline 20 & $3.15794541-0.07694650 i$ & $3.15831026-0.07693702 i$ & $3.15870213-0.07692683 i$ & $3.15912103-0.07691594 i$ \\
\hline 30 & $4.69696798-0.07696489 i$ & $4.69721326-0.07696060 i$ & $4.69747671-0.07695599 i$ & $4.69775833-0.07695107 i$ \\
\hline$\ell$ & $m M=0.34$ & $m M=0.36$ & $m M=0.38$ & $m M=0.40$ \\
\hline 0 & $0.18302899-0.05461419 i$ & $0.19355845-0.05408027 i$ & $0.20409290-0.05366384 i$ & $0.21463710-0.05334089 i$ \\
\hline 1 & $0.27635558-0.06549424 i$ & $0.28270766-0.06422074 i$ & $0.28935890-0.06302293 i$ & $0.29628643-0.06190680 i$ \\
\hline 2 & $0.41221109-0.07215671 i$ & $0.41603215-0.07143638 i$ & $0.42007211-0.07069577 i$ & $0.42432983-0.06993871 i$ \\
\hline 10 & $1.62318391-0.07669220 i$ & $1.62410605-0.07664561 i$ & $1.62508081-0.07659641 i$ & $1.62610817-0.07654462 i$ \\
\hline 20 & $3.15956693-0.07690435 i$ & $3.16003986-0.07689206 i$ & $3.16053979-0.07687908 i$ & $3.16106674-0.07686540 i$ \\
\hline 30 & $4.69805811-0.07694583 i$ & $4.69837606-0.07694027 i$ & $4.69871217-0.07693440 i$ & $4.69906645-0.07692821 i$ \\
\hline
\end{tabular}

Table 3. Dominant photon sphere modes $\left(n_{P S}=0\right)$ for massive scalar fields with $\ell=0,1,2,10,20$, and 30 in the background of a Schwarzschild-de Sitter black hole with $M^{2} \Lambda=0.04$. 


\begin{tabular}{|c|c|c|c|c|}
\hline$\ell$ & $m M=0.02$ & $m M=0.03$ & $m M=0.04$ & $m M=0.05$ \\
\hline 0 & $-0.00089412 i$ & $-0.00202588 i$ & $-0.00363772 i$ & $-0.00575958 i$ \\
\hline 1 & $-0.11631962 i$ & $-0.11767119 i$ & $-0.11958675 i$ & $-0.12209126 i$ \\
\hline 2 & $-0.23192813 i$ & $-0.23329938 i$ & $-0.23524197 i$ & $-0.23778024 i$ \\
\hline$\ell$ & $m M=0.06$ & $m M=0.07$ & $m M=0.08$ & $m M=0.09$ \\
\hline 0 & $-0.00843402 i$ & $-0.01172035 i$ & $-0.01570111 i$ & $-0.02049265 i$ \\
\hline 1 & $-0.12521929 i$ & $-0.12901740 i$ & $-0.13354774 i$ & $-0.13889355 i$ \\
\hline 2 & $-0.24094791 i$ & $-0.24479034 i$ & $-0.24936799 i$ & $-0.25476169 i$ \\
\hline$\ell$ & $m M=0.10$ & $m M=0.11$ & $m M=0.12$ & $m M=0.13$ \\
\hline 0 & $-0.02626288 i$ & $-0.03326237 i$ & $-0.04188062 i$ & $-0.05274799 i$ \\
\hline 1 & $-0.14516806 i$ & $-0.15252930 i$ & $-0.16120646 i$ & $-0.17155137 i$ \\
\hline 2 & $-0.26108116 i$ & $-0.26847912 i$ & $-0.27717645 i$ & $-0.28751105 i$ \\
\hline$\ell$ & $m M=0.14$ & $m M=0.15$ & $m M=0.16$ & $m M=0.17$ \\
\hline 0 & $-0.06689633 i$ & $-0.08585232 i$ & $-0.11152914 i$ & $-0.15564561 i$ \\
\hline 1 & $-0.18415171 i$ & $-0.20013275 i$ & $-0.22228398 i$ & $-0.26715306 i$ \\
\hline 2 & $-0.30004513 i$ & $-0.31584894 i$ & $-0.33754487 i$ & $-0.37950874 i$ \\
\hline$\ell$ & $m M=0.18$ & $m M=0.19$ & $m M=0.20$ & $m M=0.21$ \\
\hline 0 & $0.05353197-0.17154859 i$ & $0.07353052-0.16967135 i$ & $0.08727790-0.16851920 i$ & $0.09823954-0.16766383 i$ \\
\hline 1 & $0.06336411-0.28338941 i$ & $0.09146811-0.27990619 i$ & $0.11287548-0.27722512 i$ & $0.13110568-0.27524716 i$ \\
\hline 2 & $0.06076188-0.39913464 i$ & $0.08893906-0.39604938 i$ & $0.11043784-0.39339544 i$ & $0.12880897-0.39098624 i$ \\
\hline$\ell$ & $m M=0.22$ & $m M=0.23$ & $m M=0.24$ & $m M=0.25$ \\
\hline 0 & $0.10763027-0.16695588 i$ & $0.11602271-0.16633358 i$ & $0.12373171-0.16576818 i$ & $0.13094823-0.16524502 i$ \\
\hline 1 & $0.14737955-0.27403938 i$ & $0.16214487-0.27374020 i$ & $0.17539633-0.27438833 i$ & $0.18697588-0.27570348 i$ \\
\hline 2 & $0.14531497-0.38872888 i$ & $0.16056963-0.38657115 i$ & $0.17492361-0.38448131 i$ & $0.18859932-0.38243893 i$ \\
\hline$\ell$ & $m M=0.26$ & $m M=0.28$ & $m M=0.30$ & $m M=0.32$ \\
\hline 0 & $0.13779637-0.16475606 i$ & $0.15070260-0.16386364 i$ & $0.16288342-0.16306984 i$ & $0.17458166-0.16236395 i$ \\
\hline 1 & $0.19696460-0.27719964 i$ & $0.21350983-0.27958773 i$ & $0.22735961-0.28088060 i$ & $0.23974155-0.28135785 i$ \\
\hline 2 & $0.20174945-0.38043017 i$ & $0.22689872-0.37647517 i$ & $0.25104980-0.37254550 i$ & $0.27481104-0.36851568 i$ \\
\hline$\ell$ & $m M=0.34$ & $m M=0.36$ & $m M=0.38$ & $m M=0.40$ \\
\hline 0 & $0.18594576-0.16173872 i$ & $0.19707225-0.16118815 i$ & $0.20802686-0.16070674 i$ & $0.21885598-0.16028920 i$ \\
\hline 1 & $0.25124153-0.28130544 i$ & $0.26217041-0.28091663 i$ & $0.27271023-0.28031643 i$ & $0.28297559-0.27958645 i$ \\
\hline 2 & $0.29906539-0.36392925 i$ & $0.32509244-0.35591310 i$ & $0.34460718-0.34125596 i$ & $0.35774257-0.33038504 i$ \\
\hline
\end{tabular}

Table 4. Dominant dS modes $\left(n_{d S}=0\right)$ for massive scalar fields with $\ell=0,1,2,10,20$, and 30 in the background of a Schwarzschild-de Sitter black hole with $M^{2} \Lambda=0.04$. 


\begin{tabular}{|c|c|c|c|c|}
\hline$\ell$ & $m M=0.02$ & $m M=0.03$ & $m M=0.04$ & $m M=$ \\
\hline 1 & $21538154-0.24734336 i$ & $21546087-0.24716801 i$ & $.21557160-0.24692237 i$ & $0.21571337-0.24660629 i$ \\
\hline 2 & $7173556-0.23788191 i$ & $83876-$ & $37198332-0.23767614 i$ & $.37216932-0.237521$ \\
\hline 10 & $61300608-0.23133660 i$ & $61303869-0.23133156 i$ & $.61308436-0.23132451 i$ & $.61314307-0.23131544 i$ \\
\hline 20 & $15434249-0.23104400 i$ & $.15435934-0.23104268 i$ & $3.15438293-0.23104083 i$ & $3.15441326-0.23103845 i$ \\
\hline 30 & $69454512-$ & $.69455646-$ & 1.69457234 & $4.69459276-$ \\
\hline$\ell$ & $m M=0.06$ & $m M=0.07$ & $m M=0.08$ & $m M=0.09$ \\
\hline 1 & $21588561-0.24$ & $21608750-0.2457$ & $21631786-0.2452$ & $.21657503-0.2446$ \\
\hline 2 & $39683-$ & $15 i$ & & $72 i$ \\
\hline 10 & $61321482-0.23130435 i$ & $61329962-0.23129125 i$ & $1.61339747-0.23127614 i$ & $1.61350837-0.23125901 i$ \\
\hline 20 & $145032-$ & $12 i$ & $6 i$ & 50196 \\
\hline 30 & $69461771-$ & $64720-$ & $68123-$ & $471979-$ \\
\hline$\ell$ & $m M=$ & $m M=$ & $m M=$ & $m M=$ \\
\hline 1 & $1805 c 72$ & & 710010 & $73 i$ \\
\hline 2 & $37372489-$ & 37416230 & 64233 & $.37516526-$ \\
\hline 10 & $61363231-$ & $76929-$ & $1933-$ & $88240-$ \\
\hline 20 & $6598-$ & $3675-$ & $5 i$ & \\
\hline 30 & $69476290-0.2309$ & $69481053-$ & $4.69486271-0.2309$ & $4.69491942-0.230$ \\
\hline$\ell$ & $m M=$ & $m M$ & $m M$ & $m M$ \\
\hline 1 & $14812-$ & 47889 & 79209 & 07242 \\
\hline 2 & $3141-$ & $33 i$ & $1 i$ & 769299 \\
\hline 10 & $5853-$ & $14770-$ & 4991 & $62 i$ \\
\hline 20 & $98947-$ & 08719 & $49 i$ & $530284-$ \\
\hline 30 & $498067-0$ & $504646-$ & $11678-$ & $019104-0$ \\
\hline$\ell$ & $m M=$ & $m M=$ & $m M=$ & $m M=$ \\
\hline 1 & $30139-$ & 45849 & 52555 & 50065 \\
\hline 2 & $3607-$ & $2468-$ & 594 & $65 i$ \\
\hline 10 & $609348-$ & $33483-$ & 58923 & 585667 \\
\hline 20 & 42078 & $0 i$ & $1 i$ & 81501 \\
\hline 30 & $27104-$ & $35498-$ & $44345-$ & $553646-$ \\
\hline$\ell$ & $m M=$ & $m M=$ & $m M$ & $m M=$ \\
\hline 1 & $943499-$ & 50888 & 009313 & $158161-$ \\
\hline 2 & $87055-$ & 84868 & $387659-$ & $3495554-$ \\
\hline 10 & $61613716-$ & $43069-$ & 73726 & $705688-$ \\
\hline 20 & $595990-$ & $611153-$ & 626989 & $643499-$ \\
\hline 30 & $.69563400-0.2309$ & $.69573608-0.23092446 i$ & $4.69584270-0.23091886 i$ & $4.69595385-0.2309$ \\
\hline$\ell$ & $m M=$ & $m M=$ & $m M=$ & $m M=$ \\
\hline 1 & $05623-$ & $6721-$ & $14221-$ & $834770-$ \\
\hline 2 & $38608693-0.22$ & $51376-$ & $117274-$ & $408350-0.219$ \\
\hline 10 & $61738954-$ & $809400-$ & $.61885062-$ & $1.61965942-$ \\
\hline 20 & $3.15660683-0.23086673 i$ & $.15697073-0.2308382$ & $3.15736157-0.23080770 i$ & $3.15777937-0.2307$ \\
\hline 30 & $69606954-0.23090695 i$ & $69631453-$ & $4.69657767-0.2308$ & $4.69685895-0.23086$ \\
\hline$\ell$ & $m M=0.34$ & $m M=0.36$ & $m M=$ & $m M=0.40$ \\
\hline 1 & $55745578-0.18597950 i$ & $26663309-0.18245622 i$ & $0.27582463-0.17948705 i$ & $.28500951-0.1769$ \\
\hline 2 & 26960 & 075749 & 57407 & $74302-$ \\
\hline 10 & $1.62052039-0.23018019 i$ & $.62143352-0.23004031 i$ & $1.62239881-0.22989260 i$ & $1.62341626-0.22973707 i$ \\
\hline 20 & $3.15822411-0.23074026 i$ & $3.15869579-0.23070340 i$ & $3.15919442-0.23066444 i$ & $3.15972000-0.23062339 i$ \\
\hline 30 & $4.69715838-0.23084978 i$ & $4.69747595-0.23083311 i$ & $4.69781166-0.23081549 i$ & $4.69816552-0.23079692 i$ \\
\hline
\end{tabular}

Table 5. Photon sphere modes for the first overtone number $\left(n_{P S}=1\right)$ for massive scalar fields with $\ell=1,2,10,20$, and 30 in the background of a Schwarzschild-de Sitter black hole with $M^{2} \Lambda=0.04$. 


\section{Numerical values. Schwarzschild-de Sitter black holes with $M^{2} \Lambda=0.11$}

\begin{tabular}{|c|c|c|c|c|}
\hline$\ell$ & $m M=0.02$ & $m M=0.03$ & $m M=0.04$ & $m M=0.05$ \\
\hline 0 & $0.0020380076-0.0189811106 i$ & $0.0020383581-0.0189800488 i$ & $0.0020327260-0.0189784691 i$ & $0.0020144687-0.0189762687 i$ \\
\hline 1 & $0.02551665-0.00964921 i$ & $0.02554935-0.00964894 i$ & $0.02559507-0.00964856 i$ & $0.02565373-0.00964808 i$ \\
\hline 2 & $0.04618334-0.00963129 i$ & $0.04620139-0.00963121 i$ & $0.04622664-0.00963112 i$ & $0.04625909-0.00963099 i$ \\
\hline 10 & $0.20162233-0.00962298 i$ & $0.20162647-0.00962298 i$ & $0.20163225-0.00962297 i$ & $0.20163969-0.00962297 i$ \\
\hline 20 & $0.39429226-0.00962263 i$ & $0.39429437-0.00962263 i$ & $0.39429733-0.00962263 i$ & $0.39430114-0.00962263 i$ \\
\hline 30 & $0.58681792-0.00962256 i$ & $0.58681934-0.00962256 i$ & $0.58682133-0.00962256 i$ & $0.58682389-0.00962256 i$ \\
\hline$\ell$ & $m M=0.06$ & $m M=0.07$ & $m M=0.08$ & $m M=0.09$ \\
\hline 0 & $0.0019739262-0.0189732995 i$ & $0.0018966550-0.0189693514 i$ & $0.0017591920-0.0189641247 i$ & $0.0015166966-0.0189571824 i$ \\
\hline 1 & $0.02572524-0.00964751 i$ & $0.02580950-0.00964683 i$ & $0.02590639-0.00964607 i$ & $0.02601575-0.00964522 i$ \\
\hline 2 & $0.04629873-0.00963084 i$ & $0.04634552-0.00963066 i$ & $0.04639946-0.00963045 i$ & $0.04646051-0.00963022 i$ \\
\hline 10 & $0.20164878-0.00962296 i$ & $0.20165953-0.00962295 i$ & $0.20167193-0.00962294 i$ & $0.20168597-0.00962293 i$ \\
\hline 20 & $0.39430579-0.00962262 i$ & $0.39431128-0.00962262 i$ & $0.39431762-0.00962262 i$ & $0.39432481-0.00962262 i$ \\
\hline 30 & $0.58682701-0.00962256 i$ & $0.58683071-0.00962256 i$ & $0.58683497-0.00962256 i$ & $0.58683979-0.00962256 i$ \\
\hline$\ell$ & $m M=0.10$ & $m M=0.11$ & $m M=0.12$ & $m M=0.13$ \\
\hline 0 & $0.0010460297-0.0189478654 i$ & $-0.0181039644 i$ & $-0.0171016030 i$ & $-0.0162275285 i$ \\
\hline 1 & $0.02613743-0.00964429 i$ & $0.02627127-0.00964329 i$ & $0.02641707-0.00964226 i$ & $0.02657464-0.00964110 i$ \\
\hline 2 & $0.04652865-0.00962996 i$ & $0.04660385-0.00962967 i$ & $0.04668607-0.00962937 i$ & $0.04677528-0.00962904 i$ \\
\hline 10 & $0.20170168-0.00962292 i$ & $0.20171903-0.00962290 i$ & $0.20173803-0.00962289 i$ & $0.20175868-0.00962287 i$ \\
\hline 20 & $0.39433284-0.00962261 i$ & $0.39434171-0.00962261 i$ & $0.39435143-0.00962261 i$ & $0.39436110-0.00962260 i$ \\
\hline 30 & $0.58684519-0.00962255 i$ & $0.58685115-0.00962255 i$ & $0.58685769-0.00962255 i$ & $0.58686479-0.00962255 i$ \\
\hline$\ell$ & $m M=0.14$ & $m M=0.15$ & $m M=0.16$ & $m M=0.17$ \\
\hline 0 & $-0.0152852132 i$ & $-0.0141564333 i$ & $-0.01258354445 i$ & $0.00189593-0.00973749 i$ \\
\hline 1 & $0.02674378-0.00963992 i$ & $0.02692425-0.00963869 i$ & $0.02711585-0.00963742 i$ & $0.02731832-0.00963611 i$ \\
\hline 2 & $0.04687144-0.00962868 i$ & $0.04697450-0.00962831 i$ & $0.04708443-0.00962791 i$ & $0.04720116-0.00962750 i$ \\
\hline 10 & $0.20178099-0.00962285 i$ & $0.20180494-0.00962283 i$ & $0.20183054-0.00962281 i$ & $0.20185779-0.00962279 i$ \\
\hline 20 & $0.39437341-0.00962260 i$ & $0.39438567-0.00962259 i$ & $0.39439877-0.00962259 i$ & $0.39441271-0.00962258 i$ \\
\hline 30 & $0.58687245-0.00962255 i$ & $0.58688069-0.00962254 i$ & $0.58688949-0.00962254 i$ & $0.58683071-0.00962256 i$ \\
\hline$\ell$ & $m M=0.18$ & $m M=0.19$ & $m M=0.20$ & $m M=0.21$ \\
\hline 0 & $0.00396067-0.00971436 i$ & $0.00532622-0.00969559 i$ & $0.00645433-0.00968015 i$ & $0.00745450-0.00966731 i$ \\
\hline 1 & $0.02753144-0.00963478 i$ & $0.02775495-0.00963343 i$ & $0.02798862-0.00963206 i$ & $0.02823218-0.00963068 i$ \\
\hline 2 & $0.04732466-0.00962707 i$ & $0.04745487-0.00962662 i$ & $0.04759173-0.00962615 i$ & $0.04773520-0.00962566 i$ \\
\hline 10 & $0.20188669-0.00962277 i$ & $0.20191723-0.00962274 i$ & $0.20194942-0.00962272 i$ & $0.20198326-0.00962269 i$ \\
\hline 20 & $0.39442750-0.00962257 i$ & $0.39444314-0.00962257 i$ & $0.39445961-0.00962256 i$ & $0.39447694-0.00962255 i$ \\
\hline 30 & $0.58690880-0.00962254 i$ & $0.58691931-0.00962253 i$ & $0.58693039-0.00962253 i$ & $0.58694203-0.00962253 i$ \\
\hline$\ell$ & $m M=0.22$ & $m M=0.23$ & $m M=0.24$ & $m M=0.25$ \\
\hline 0 & $0.00837329-0.00965655 i$ & $0.00923542-0.00964746 i$ & $0.01005577-0.00963973 i$ & $0.01084402-0.00963313 i$ \\
\hline 1 & $0.02848538-0.00962930 i$ & $0.02874797-0.00962792 i$ & $0.02901970-0.00962654 i$ & $0.02930030-0.00962518 i$ \\
\hline 2 & $0.04788520-0.00962517 i$ & $0.04804168-0.00962465 i$ & $0.04820458-0.00962413 i$ & $0.04837383-0.00962360 i$ \\
\hline 10 & $0.20201874-0.00962266 i$ & $0.20205586-0.00962263 i$ & $0.20209463-0.00962260 i$ & $0.20213503-0.00962257 i$ \\
\hline 20 & $0.39449510-0.00962255 i$ & $0.39451412-0.00962254 i$ & $0.39453397-0.00962253 i$ & $0.39455467-0.00962252 i$ \\
\hline 30 & $0.58695424-0.00962252 i$ & $0.58696702-0.00962252 i$ & $0.58698036-0.00962252 i$ & $0.58699427-0.00962251 i$ \\
\hline$\ell$ & $m M=0.26$ & $m M=0.28$ & $m M=0.30$ & $m M=0.32$ \\
\hline 0 & $0.01160687-0.00962747 i$ & $0.01307445-0.00961838 i$ & $0.01448450-0.00961154 i$ & $0.01585274-0.00960634 i$ \\
\hline 1 & $0.02958954-0.00962383 i$ & $0.03019290-0.00962118 i$ & $0.03082781-0.00961862 i$ & $0.03149238-0.00961618 i$ \\
\hline 2 & $0.04854937-0.00962305 i$ & $0.04891903-0.00962193 i$ & $0.04931301-0.00962078 i$ & $0.04973074-0.00961961 i$ \\
\hline 10 & $0.20217708-0.00962254 i$ & $0.20226610-0.00962247 i$ & $0.20236167-0.00962239 i$ & $0.20246377-0.00962231 i$ \\
\hline 20 & $0.39457621-0.00962251 i$ & $0.39462183-0.00962250 i$ & $0.39467082-0.00962248 i$ & $0.39472318-0.00962245 i$ \\
\hline 30 & $0.58700875-0.00962251 i$ & $0.58703942-0.00962250 i$ & $0.58707235-0.00962249 i$ & $0.58710755-0.00962248 i$ \\
\hline$\ell$ & $m M=0.34$ & $m M=0.36$ & $m M=0.38$ & $m M=0.40$ \\
\hline 0 & $0.01718939-0.00960235 i$ & $0.01850145-0.00959927 i$ & $0.01979391-0.00959688 i$ & $0.02107044-0.00959501 i$ \\
\hline 1 & $0.03218476-0.00961386 i$ & $0.03290322-0.00961167 i$ & $0.03364608-0.00960962 i$ & $0.03441178-0.00960771 i$ \\
\hline 2 & $0.05017161-0.00961843 i$ & $0.05063505-0.00961724 i$ & $0.05112043-0.00961605 i$ & $0.05162713-0.00961487 i$ \\
\hline 10 & $0.20257241-0.00962223 i$ & $0.20268758-0.00962214 i$ & $0.20280925-0.00962204 i$ & $0.20293742-0.00962194 i$ \\
\hline 20 & $0.39477891-0.00962243 i$ & $0.39483802-0.00962241 i$ & $0.39490049-0.00962238 i$ & $0.39496632-0.00962236 i$ \\
\hline 30 & $0.58714502-0.00962247 i$ & $0.58718476-0.00962246 i$ & $0.58722677-0.00962245 i$ & $0.58727105-0.00962244 i$ \\
\hline
\end{tabular}

Table 6. Fundamental quasinormal frequencies $(n=0)$ for massive scalar fields with $\ell=0,1,2$, 10,20 , and 30 in the background of a Schwarzschild-de Sitter black hole with $M^{2} \Lambda=0.11$. 


\section{Numerical values. Schwarzschild-AdS black holes}

\begin{tabular}{|c|c|c|c|c|}
\hline$\ell$ & $m M=0$ & $m M=0.05$ & $m M=0.10$ & $m M=0.15$ \\
\hline 0 & $18.60702097-26.64177595 i$ & $18.61223099-26.65137963 i$ & $18.62784397-26.68016019 i$ & $18.65380900-26.72802663 i$ \\
1 & $18.68948190-26.61845039 i$ & $18.69467400-26.62805066 i$ & $18.71023335-26.65682099 i$ & $18.73610934-26.70467047 i$ \\
2 & $18.85273204-26.57247886 i$ & $18.85788929-26.58207228 i$ & $18.87334427-26.61082210 i$ & $18.89904696-26.65863754 i$ \\
10 & $22.51253455-25.61606706 i$ & $22.51707726-25.62548491 i$ & $22.53069166-25.65370904 i$ & $22.55333682-25.70065162 i$ \\
20 & $30.21903865-24.02953567 i$ & $30.22286541-24.03855067 i$ & $30.23433465-24.06556806 i$ & $30.25341348-24.11050556 i$ \\
30 & $39.09826401-22.71395892 i$ & $39.10165741-22.72256722 i$ & $39.11182789-22.74836610 i$ & $39.12874649-22.79127794 i$ \\
\hline$\ell$ & $m M=0.20$ & $m M=0.25$ & $m M=0.30$ & $m M=0.35$ \\
\hline 0 & $18.69004218-26.79482897 i$ & $18.73642795-26.88036058 i$ & $18.79282102-26.98436146 i$ & $18.85904854-27.10652214 i$ \\
1 & $18.77221856-26.77144920 i$ & $18.81844615-26.85695073 i$ & $18.87464766-26.96091523 i$ & $18.94065129-27.08303345 i$ \\
2 & $18.93491491-26.72536893 i$ & $18.98083460-26.81081010 i$ & $19.03666324-26.91470159 i$ & $19.10223099-27.03673460 i$ \\
10 & $22.58494526-25.76616790 i$ & $22.62542404-25.85005843 i$ & $22.67465618-25.95207222 i$ & $22.73250244-26.07191052 i$ \\
20 & $30.28004766-24.17322743 i$ & $30.31416248-24.25354675 i$ & $30.35566387-24.35122822 i$ & $30.40443990-24.46599175 i$ \\
30 & $39.15236549-22.85117479 i$ & $39.18261910-22.92788037 i$ & $39.21942456-23.02117280 i$ & $39.26268334-23.13078800 i$ \\
\hline$\ell$ & $m M=0.40$ & $m M=0.45$ & $m M=0.50$ & $m M=0.60$ \\
\hline 0 & $18.93491276-27.24648824 i$ & $19.02019383-27.40386537 i$ & $19.11465281-27.57822445 i$ & $19.33007160-27.97603061 i$ \\
1 & $19.01626044-27.22295125 i$ & $19.10125653-27.38027456 i$ & $19.19540202-27.55457456 i$ & $19.41011414-27.95224815 i$ \\
2 & $19.17734349-27.17655550 i$ & $19.26178462-27.33377075 i$ & $19.35531951-27.50795215 i$ & $19.56865488-27.90535950 i$ \\
10 & $22.79880337-26.20923117 i$ & $22.87338152-26.36365336 i$ & $22.95604382-26.53476268 i$ & $23.14478480-26.92524821 i$ \\
20 & $30.46036235-24.59751656 i$ & $30.52328849-24.74544559 i$ & $30.59306299-24.90939025 i$ & $30.75248432-25.28364347 i$ \\
30 & $39.31228260-23.25642346 i$ & $39.36809675-23.39774250 i$ & $39.42998910-23.55437869 i$ & $39.57141652-23.91201550 i$ \\
\hline
\end{tabular}

Table 7. Fundamental quasinormal frequencies $\omega M(n=0)$ of massive scalar fields with $\ell=0,1$, 2, 10, 20,30 in the background of Schwarzschild-AdS black holes with $M^{2} \Lambda=-765075$, or $r_{H} / R=$ 10 , where $R$ is the AdS radius.

\begin{tabular}{|c|c|c|c|c|}
\hline$\ell$ & $m M=0$ & $m M=0.05$ & $m M=0.10$ & $m M=0.15$ \\
\hline 0 & $1.46486212-1.14108905 i$ & $1.46604026-1.14240948 i$ & $1.46956344-1.14635875 i$ & $1.47539846-1.15290143 i$ \\
1 & $1.81421829-1.01340851 i$ & $1.81524513-1.01466010 i$ & $1.81831636-1.01840398 i$ & $1.82340447-1.02460801 i$ \\
2 & $2.30384515-0.87938138 i$ & $2.30476262-0.88053246 i$ & $2.30750672-0.88397613 i$ & $2.31205302-0.88968420 i$ \\
10 & $6.88156796-0.36773408 i$ & $6.88230126-0.36838601 i$ & $6.88449386-0.37033762 i$ & $6.88812433-0.37357663 i$ \\
20 & $12.75994862-0.13788816 i$ & $12.76068546-0.13825105 i$ & $12.76288803-0.13933865 i$ & $12.76653284-0.14114771 i$ \\
30 & $18.64042583-0.03916775 i$ & $18.64122199-0.03933376 i$ & $18.64360088-0.03983262 i$ & $18.64753421-0.04066680 i$ \\
\hline$\ell$ & $m M=0.20$ & $m M=0.25$ & $m M=0.30$ & $m M=0.35$ \\
\hline 0 & $1.48349180-1.16198028 i$ & $1.49377204-1.17351886 i$ & $1.50615298-1.18742468 i$ & $1.52053704-1.20359279 i$ \\
1 & $1.83046511-1.03322029 i$ & $1.83943909-1.04417142 i$ & $1.85025488-1.05737742 i$ & $1.86283146-1.07274292 i$ \\
2 & $2.31836207-0.89761108 i$ & $2.32638124-0.90769585 i$ & $2.33604694-0.91986473 i$ & $2.34728717-0.93403383 i$ \\
10 & $6.89315806-0.37808311 i$ & $6.89954897-0.38383036 i$ & $6.90724147-0.39078590 i$ & $6.91617272-0.39891264 i$ \\
20 & $12.77158207-0.14367296 i$ & $12.77798542-0.14690720 i$ & $12.78568235-0.15084152 i$ & $12.79460465-0.15546547 i$ \\
30 & $18.65297651-0.04184025 i$ & $18.65986742-0.04335825 i$ & $18.66813447-0.04522712 i$ & $18.67769632-0.04745393 i$ \\
\hline$\ell$ & $m M=0.40$ & $m M=0.45$ & $m M=0.50$ & $m M=0.60$ \\
\hline 0 & $1.53681870-1.22190939 i$ & $1.55488782-1.24225525 i$ & $1.57463255-1.26450882 i$ & $1.61870707-1.31425634 i$ \\
1 & $1.8770811-1.09016436 i$ & $1.89291216-1.10953318 i$ & $1.91023135-1.13073850 i$ & $1.94896518-1.17821766 i$ \\
2 & $2.36002403-0.95011207 i$ & $2.37417610-0.96800382 i$ & $2.38966064-0.98761134 i$ & $2.42429982-1.03158437 i$ \\
10 & $6.92627490-0.40817005 i$ & $6.93747734-0.41851531 i$ & $6.94970842-0.42990431 i$ & $6.97697454-0.45563597 i$ \\
20 & $12.80467891-0.16076733 i$ & $12.81582892-0.16673431 i$ & $12.82797782-0.17335278 i$ & $12.85497129-0.18848669 i$ \\
30 & $18.68846579-0.05004619 i$ & $18.70035289-0.05301153 i$ & $18.71326738-0.05635742 i$ & $18.74182840-0.06421819 i$ \\
\hline
\end{tabular}

Table 8. Fundamental quasinormal frequencies $\omega M(n=0)$ of massive scalar fields with $\ell=0,1$, 2, 10, 20, 30 in the background of Schwarzschild-AdS black holes with $M^{2} \Lambda=-1.0$. 


\begin{tabular}{|c|c|c|c|c|}
\hline$\ell$ & $m M=0$ & $m M=0.05$ & $m M=0.10$ & $m M=0.15$ \\
\hline 0 & $0.11234408-0.00253387 i$ & $0.12751958-0.00347144 i$ & $0.15948982-0.00612361 i$ & $0.19628098-0.01039618 i$ \\
1 & $0.15689207-0.00002564 i$ & $0.17300519-0.00004312 i$ & $0.20700088-0.00011796 i$ & $0.24584844-0.00033499 i$ \\
2 & $0.19924295-7.07258 * 10^{-8} i$ & $0.21579949-1.3258 * 10^{-7} i$ & $0.25094984-4.4974 * 10^{-7} i$ & $0.29155767-1.6262 * 10^{-6} i$ \\
\hline$\ell$ & $m M=0.20$ & $m M=0.25$ & $m M=0.30$ & $m M=0.35$ \\
\hline 0 & $0.23466144-0.01622251 i$ & $0.27386451-0.02346464 i$ & $0.31372182-0.03195017 i$ & $0.35422112-0.04150833 i$ \\
1 & $0.28556635-0.00088357 i$ & $0.32491192-0.00208114 i$ & $0.36356073-0.00429995 i$ & $0.40161853-0.00781653 i$ \\
2 & $0.33367650-5.62773 * 10^{-6} i$ & $0.37605040-0.00001838 i$ & $0.41815083-0.00005661 i$ & $0.45967430-0.00016397 i$ \\
\hline
\end{tabular}

Table 9. Fundamental quasinormal frequencies $\omega M(n=0)$ of massive scalar fields with $\ell=0,1,2$ in the background of Schwarzschild-AdS black holes with $M^{2} \Lambda=-0.005$.

\section{Acknowledgments}

We thank the referee for his/her careful review of the manuscript and his/her valuable comments and suggestions which helped us to improve the manuscript considerably. P.A.G. acknowledges the hospitality of the Universidad de La Serena where part of this work was undertaken. Y.V. acknowledge support by the Dirección de Investigación y Desarrollo de la Universidad de La Serena, Grant No. PR18142.

Open Access. This article is distributed under the terms of the Creative Commons Attribution License (CC-BY 4.0), which permits any use, distribution and reproduction in any medium, provided the original author(s) and source are credited.

\section{References}

[1] T. Regge and J.A. Wheeler, Stability of a Schwarzschild singularity, Phys. Rev. 108 (1957) 1063 [INSPIRE].

[2] F.J. Zerilli, Gravitational field of a particle falling in a Schwarzschild geometry analyzed in tensor harmonics, Phys. Rev. D 2 (1970) 2141 [INSPIRE].

[3] K.D. Kokkotas and B.G. Schmidt, Quasinormal modes of stars and black holes, Living Rev. Rel. 2 (1999) 2 [gr-qc/9909058] [INSPIRE].

[4] H.-P. Nollert, Quasinormal modes: the characteristic 'sound' of black holes and neutron stars, Class. Quant. Grav. 16 (1999) R159 [INSPIRE].

[5] R.A. Konoplya and A. Zhidenko, Quasinormal modes of black holes: From astrophysics to string theory, Rev. Mod. Phys. 83 (2011) 793 [arXiv: 1102.4014] [INSPIRE].

[6] E. Berti, V. Cardoso and A.O. Starinets, Quasinormal modes of black holes and black branes, Class. Quant. Grav. 26 (2009) 163001 [arXiv:0905.2975] [INSPIRE].

[7] LIGO ScIEnTIFIC and VIRGO collaborations, Observation of Gravitational Waves from a Binary Black Hole Merger, Phys. Rev. Lett. 116 (2016) 061102 [arXiv:1602.03837] [INSPIRE].

[8] LIGO SCIENTIFIC and VIRGO collaborations, Tests of general relativity with GW150914, Phys. Rev. Lett. 116 (2016) 221101 [Erratum ibid. 121 (2018) 129902] [arXiv:1602.03841] [INSPIRE]. 
[9] R. Konoplya and A. Zhidenko, Detection of gravitational waves from black holes: Is there a window for alternative theories?, Phys. Lett. B 756 (2016) 350 [arXiv:1602.04738] [INSPIRE].

[10] R.A. Konoplya and A.V. Zhidenko, Decay of massive scalar field in a Schwarzschild background, Phys. Lett. B 609 (2005) 377 [gr-qc/0411059] [INSPIRE].

[11] R.A. Konoplya and A. Zhidenko, Stability and quasinormal modes of the massive scalar field around Kerr black holes, Phys. Rev. D 73 (2006) 124040 [gr-qc/0605013] [INSPIRE].

[12] S.R. Dolan, Instability of the massive Klein-Gordon field on the Kerr spacetime, Phys. Rev. $D 76$ (2007) 084001 [arXiv:0705.2880] [INSPIRE].

[13] O.J. Tattersall and P.G. Ferreira, Quasinormal modes of black holes in Horndeski gravity, Phys. Rev. D 97 (2018) 104047 [arXiv: 1804.08950] [InSPIRE].

[14] M. Lagos, P.G. Ferreira and O.J. Tattersall, Anomalous decay rate of quasinormal modes, Phys. Rev. D 101 (2020) 084018 [arXiv:2002.01897] [INSPIRE].

[15] P.R. Brady, C.M. Chambers, W. Krivan and P. Laguna, Telling tails in the presence of a cosmological constant, Phys. Rev. D 55 (1997) 7538 [gr-qc/9611056] [INSPIRE].

[16] P.R. Brady, C.M. Chambers, W.G. Laarakkers and E. Poisson, Radiative falloff in Schwarzschild-de Sitter space-time, Phys. Rev. D 60 (1999) 064003 [gr-qc/9902010] [INSPIRE].

[17] T.R. Choudhury and T. Padmanabhan, Quasinormal modes in Schwarzschild-deSitter space-time: A Simple derivation of the level spacing of the frequencies, Phys. Rev. D 69 (2004) 064033 [gr-qc/0311064] [INSPIRE].

[18] D.-P. Du, B. Wang and R.-K. Su, Quasinormal modes in pure de Sitter space-times, Phys. Rev. D 70 (2004) 064024 [hep-th/0404047] [INSPIRE].

[19] C. Molina, Quasinormal modes of d-dimensional spherical black holes with near extreme cosmological constant, Phys. Rev. D 68 (2003) 064007 [gr-qc/0304053] [INSPIRE].

[20] C. Molina, D. Giugno, E. Abdalla and A. Saa, Field propagation in de Sitter black holes, Phys. Rev. D 69 (2004) 104013 [gr-qc/0309079] [InSPIRE].

[21] J.M. Maldacena, The Large $N$ limit of superconformal field theories and supergravity, Int. J. Theor. Phys. 38 (1999) 1113 [hep-th/9711200] [INSPIRE].

[22] O. Aharony, S.S. Gubser, J.M. Maldacena, H. Ooguri and Y. Oz, Large N field theories, string theory and gravity, Phys. Rept. 323 (2000) 183 [hep-th/9905111] [InSPIRE].

[23] G.T. Horowitz and V.E. Hubeny, Quasinormal modes of AdS black holes and the approach to thermal equilibrium, Phys. Rev. D 62 (2000) 024027 [hep-th/9909056] [INSPIRE].

[24] J.S.F. Chan and R.B. Mann, Scalar wave falloff in asymptotically anti-de Sitter backgrounds, Phys. Rev. D 55 (1997) 7546 [gr-qc/9612026] [INSPIRE].

[25] H. Otsuki and T. Futamase, Gravitational perturbation of Schwarzschild-de Sitter spacetime and its quasi-normal modes, Prog. Theor. Phys. 85 (1991) 771.

[26] J.P. Boyd, Chebyshev and Fourier Spectral Methods, Dover Books on Mathematics, Dover Publications, Mineola, NY, second ed. (2001).

[27] S.I. Finazzo, R. Rougemont, M. Zaniboni, R. Critelli and J. Noronha, Critical behavior of non-hydrodynamic quasinormal modes in a strongly coupled plasma, JHEP 01 (2017) 137 [arXiv: 1610.01519] [INSPIRE]. 
[28] P.A. González, E. Papantonopoulos, J. Saavedra and Y. Vásquez, Superradiant Instability of Near Extremal and Extremal Four-Dimensional Charged Hairy Black Hole in anti-de Sitter Spacetime, Phys. Rev. D 95 (2017) 064046 [arXiv:1702.00439] [INSPIRE].

[29] P.A. Gonzalez, Y. Vasquez and R.N. Villalobos, Perturbative and nonperturbative fermionic quasinormal modes of Einstein-Gauss-Bonnet-AdS black holes, Phys. Rev. D 98 (2018) 064030 [arXiv: 1807.11827] [INSPIRE].

[30] R. Bécar, P.A. González, E. Papantonopoulos and Y. Vásquez, Quasinormal modes of three-dimensional rotating Hořava AdS black hole and the approach to thermal equilibrium, Eur. Phys. J. C 80 (2020) 600 [arXiv:1906.06654] [INSPIRE].

[31] A. Aragón, R. Bécar, P.A. González and Y. Vásquez, Perturbative and nonperturbative quasinormal modes of 4D Einstein-Gauss-Bonnet black holes, arXiv:2004.05632 [INSPIRE].

[32] O.J.C. Dias, P. Figueras, R. Monteiro, J.E. Santos and R. Emparan, Instability and new phases of higher-dimensional rotating black holes, Phys. Rev. D 80 (2009) 111701 [arXiv: 0907.2248] [INSPIRE].

[33] F. Mellor and I. Moss, Stability of Black Holes in de Sitter Space, Phys. Rev. D 41 (1990) 403 [INSPIRE].

[34] I.G. Moss and J.P. Norman, Gravitational quasinormal modes for anti-de Sitter black holes, Class. Quant. Grav. 19 (2002) 2323 [gr-qc/0201016] [INSPIRE].

[35] A. Zhidenko, Quasinormal modes of Schwarzschild de Sitter black holes, Class. Quant. Grav. 21 (2004) 273 [gr-qc/0307012] [INSPIRE].

[36] R.A. Konoplya and A. Zhidenko, High overtones of Schwarzschild-de Sitter quasinormal spectrum, JHEP 06 (2004) 037 [hep-th/0402080] [INSPIRE].

[37] A. Jansen, Overdamped modes in Schwarzschild-de Sitter and a Mathematica package for the numerical computation of quasinormal modes, Eur. Phys. J. Plus 132 (2017) 546 [arXiv: 1709.09178] [INSPIRE].

[38] V. Cardoso, J.L. Costa, K. Destounis, P. Hintz and A. Jansen, Quasinormal modes and Strong Cosmic Censorship, Phys. Rev. Lett. 120 (2018) 031103 [arXiv:1711.10502] [INSPIRE].

[39] B. Toshmatov and Z. Stuchlík, Slowly decaying resonances of massive scalar fields around Schwarzschild-de Sitter black holes, Eur. Phys. J. Plus 132 (2017) 324 [arXiv:1707.07419] [INSPIRE].

[40] A. Lopez-Ortega, Quasinormal modes of D-dimensional de Sitter spacetime, Gen. Rel. Grav. 38 (2006) 1565 [gr-qc/0605027] [INSPIRE].

[41] J. Grain and A. Barrau, A WKB approach to scalar fields dynamics in curved space-time, Nucl. Phys. B $\mathbf{7 4 2}$ (2006) 253 [hep-th/0603042] [INSPIRE].

[42] G. Festuccia and H. Liu, A Bohr-Sommerfeld quantization formula for quasinormal frequencies of AdS black holes, Adv. Sci. Lett. 2 (2009) 221 [arXiv:0811.1033] [INSPIRE].

[43] E. Berti, V. Cardoso and P. Pani, Breit-Wigner resonances and the quasinormal modes of anti-de Sitter black holes, Phys. Rev. D 79 (2009) 101501 [arXiv:0903.5311] [InSPIRE].

[44] B. Mashhoon, Quasi-normal modes of a black hole, in Proceedings of the Third Marcel Grossmann Meeting on General Relativity, Part A, pp. 599-608 (1983). 
[45] B.F. Schutz and C.M. Will, Black hole normal modes: a semianalytic approach, Astrophys. J. Lett. 291 (1985) L33 [INSPIRE].

[46] S. Iyer and C.M. Will, Black Hole Normal Modes: A WKB Approach. 1. Foundations and Application of a Higher Order WKB Analysis of Potential Barrier Scattering, Phys. Rev. D 35 (1987) 3621 [INSPIRE].

[47] R.A. Konoplya, Quasinormal behavior of the d-dimensional Schwarzschild black hole and higher order WKB approach, Phys. Rev. D 68 (2003) 024018 [gr-qc/0303052] [INSPIRE].

[48] J. Matyjasek and M. Opala, Quasinormal modes of black holes. The improved semianalytic approach, Phys. Rev. D 96 (2017) 024011 [arXiv: 1704.00361] [InSPIRE].

[49] R.A. Konoplya, A. Zhidenko and A.F. Zinhailo, Higher order WKB formula for quasinormal modes and grey-body factors: recipes for quick and accurate calculations, Class. Quant. Grav. 36 (2019) 155002 [arXiv: 1904.10333] [INSPIRE].

[50] Y. Hatsuda, Quasinormal modes of black holes and Borel summation, Phys. Rev. D 101 (2020) 024008 [arXiv: 1906.07232] [INSPIRE].

[51] A. Aragón, P.A. González, E. Papantonopoulos and Y. Vásquez, Quasinormal modes and their anomalous behavior for black holes in $f(R)$ gravity, arXiv:2005.11179 [INSPIRE].

[52] K. Destounis, R.D.B. Fontana, F.C. Mena and E. Papantonopoulos, Strong Cosmic Censorship in Horndeski Theory, JHEP 10 (2019) 280 [arXiv:1908.09842] [INSPIRE].

[53] V. Cardoso, J.L. Costa, K. Destounis, P. Hintz and A. Jansen, Strong cosmic censorship in charged black-hole spacetimes: still subtle, Phys. Rev. D 98 (2018) 104007 [arXiv: 1808.03631] [INSPIRE]. 